\title{
Karl-Jürgen Bieback Leistungsabbau und Strukturwandel im Sozialrecht
}

Kaum ein anderer Rechtsbereich ist seit der Krise 1974 ff. so stark verändert worden wie das Sozialrecht. Dic Änderungen und Kürzungen im Soziallcistungssystem begannen bald nach Ausbruch der Krise 1974/75 bei damals mehr als I Mio. Arbeirslosen vor allem im Bereich der Arbeitsfördenung und des BAföG durch dic Haushaltsstrukturgeserze vom Dezember 1975. Als sich die Krise dann 1976/77 in der Rentenversicherung ( $R V$ ) auswirkte, gab es mit den 20. und 21. Rentenanpassungsgeserzen von 1977 und 1978 weitgehende Einschnitre in der RV, die z. T. auch zu Lasten der gesetzlichen Krankenversicherung (KV) und der damals wieder besser dastehenden Arbeitslosenversicherung (ArblV) gingen, während mir dem s. Änderungsgeserz zum AFG von 1979 auch einige der früheren Kürzungen wieder rückgängig gemacht wurden. Die vor allem auch strukturell durch die politische Organisarion des Markres für Gesundheicsleistungen (Dominanz der Anbietcr, der Kassenärzte und der Krankenhäuser) verursachten Kostensteigerungen in der KV wurden durch Gesetz von 1977 mit der Einführung von Koordinations- und Kontrollinstrumenten der Globalstcuerung beantwortet (konzertiere Aktion, Richrlinien für die ärzlichc Verordnungstärigkeit). Auf die sich ab 198 I wieder verschärfende Krise mir über 2 Mio. Arbeitslosen wurde mit den schwersten Kürzungen in allen Sozialleistungsbereichen durch dic Konsolidierungs- und Haushaltsstrukturgeserze $198 \mathrm{1}$ (für 1982) sowie die Haushaltsbcgleirgesetze 1983 und 1984 rcagiert. Yhr Netcoumverteilungs- und Kürzungsvolumen wird für $1982-85$ auf insgesame 2 ro Mrd. DM geschätzr'

\section{Ursachen der Kürzungen und politische Konzepionen}

1.1. Der Anlaß dieser Kürzungen liegr in den Problemen der Finanzen der SV: 2,3 Mio. Arbeitslose führen zu Beicragsausfällen von 2,8 Mrd. DM in dcr KV und 8,4 Mrd. DM in der RV jährlich'; pro 100000 Arbeirslose muß die Bundesanstalt für Arbeit ca. 1,2 Mrd. DM an zusätzlichen Ausgaben jährlich aufbringen, während sie ca. 0, 19 Mrd. DM Mindereinnahmen hat. So wurden die Kosten der Arbeirslosigkeir in den öffentlichen Haushalten für 1982 auf über 40 Mrd. DM geschäızr ${ }^{3}$.

Die ca. $\{24 \mathrm{Mrd}$. DM an Leistungen des Sozialbudgers 1982 können nicht über die

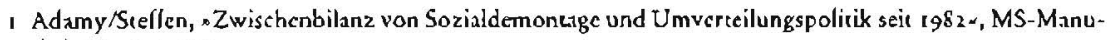
skript Januar 1984

2 Autorengemeinschaft, MiuAB 1983, S. 16.

3 Adamy/Steffen, Finanzicrungsprobleme der SV in der Wintschaftskrise. Sozialer Fonschritc 1982, S. $206 \mathrm{Hf}$.
} 
Ansammlung, "Scharzbildung “ von Kapical, sondern nur aus dem jeweiligen Sozia]produkt der Gegenwart gedeckt werden. Während in der Krise die Einnabmen des Sozialleistungssystems aus Bciträgen und Steuern zurückgehen, wachsen die Ausgabenbelastungen durch Arbeicsiosigkcit, frühzeizige Verrentung etc.

1.2. Der Abhängigkeir von der nationalen und internationalen Encwicklung der Konjunktur und ökonomischen Struktur kann sich zwar kein staaclicher Leistungsbereich entziehen, jedoch wird die spezifische Form der Krisenbewältigung im Sozialleiscungssystem in der Bundesrepublik stark bestimmt von ihrem speziellen Typus der Sozialstaarlichkeit. Der Sozialscaat der Bundesrepublik verhält sich zu seiner ökonomisch-gesellschaftlichen Basis vornehmlich reakriv und kompensatorisch, er lenkt den Marktprozeß meist nur global und indirekt über markrwirtschaftlichc Anrcize; der öffencliche Wirtschaftssektor, der direke zur politischen Steuerung eingesetzt werden könnte, ist nur in Teilbereichen ausgedehnc (Energie, Transport) und selbst darn noch den privarwirtschaftlichen Interessen zu- und untergeordnet. Seine Mittel zur Gestaltung und Steuerung schöpft der Staatsapparat über die Sreuern, Abgaben und Beiträge; er knüptr insoweit als "Steuerstastu nur an das abstrakte Ergebnis der kapitalistischen Wertproduktion an und bleibt deshalb vom Zyklus und der Strukcur der Kapicalakkumulation ablä̈ngig.

Auch in der Krise ist dieser Typus des reaktiven, kompensatorischen Sozialstaates nicht in Frage gestellt worden, wenn auch die beiden Hauptströmungen der sozialpolitischen Krisenbewälrigung das Dilemma von Steuer- und Sozialstaat deutlich zum Ansatzpunkt ihrer Vorschläge machen.

1.3. Die konservacive Lösung' will die sraatliche Abgabenquore senken und die konsumiven Staatsaufgaben (Gchälter der Staacsbediensteten, sozialstaatlichc Leistungen) kürzen und statidessen die investiven Staatsaufgaben (Intrastruktur) verstärken. Speziell wird gerade der Sozialstaat und das System der Sozialleistungen selbst für die Krise verantwortich gemacht. Dic Sozialgesetze würden die ökonomischen Anpassungsmechanismen des Marktes zurückdrängen.

Damir Leistung wieder attraktiv wird, müßten vor allem die Lohnersatzleistungen an Arbeitsfähige gesenkt und die Anspruchsinflacion abgebaut werden. Dic Verancwortung des Einzelnen für sein ökonomisches und soziales Schicksal sei zu betonen (insbcsondere die Verpflichrung der Arbeitslosen, jede Arbeit anzunehmen) wie die sozialen Risiken und ihre Beruälrigung stärker dem Einzelnen überlassen wcrden sollten. Zugleich wird die autoritäre Form des Sozialscaats, die Kontrollc des Leiscungsbezugs und des Bedaris, ausgebaut. Zwangsläufig führt dies zu einer Marginalisicrung und Ausgrenzung der nichtleisıungşähigen und -bereiten Teile der Bevölkerung.

Während dic konservative Doktrin statr der nationalen und internationalen Entwicklung der Kapitalakkumulation gleichsam das Sozialleistungssystem selber zur Krisenursache erklärt, konzencrien sich die reformonientierte Richtung darauf, die Wirkungen der ökonomischen Krise auf das Sozialleistungssystem zu analysieren und daraus Forderunged für eine Reform des Sozialieistungssystems abzuleiten. Wenn auch in stark unterschiedlicher Akzentuierung im einzelnen, so gingen schon reche früh die Gewerkschaften des DGB wie auch die politische Programmatik

4 Hicrzu allg: U. K. PreuB, Rechisstaat - Steucrstaat - Sozialstaat, eine Problemskizze, in: Abendroth U. 1.. Ordnungsmacht?, 1981, S. 46 ff.; Groth, Dic Krisc der Sozialversicherungen, XJ 1977, S. I If.

; BDA (Hrsg.), Soxiale Sicherung in der Zukunf: (1982); Soziale Siclerung im Umbruch (1983); Adamy/Steffen. Sozidabbau und Umverceilung in der Wirschaftskrise, WSl-Mir. 198 , 5.603 ff., Reidegeld, Sozialpolitik un W/andepunkt ..., Soziale Sicherheir 1983. S. 16, II.; Zachen, Hinergtund und Perspektiven der $*$ Gegenreform im Arbcitsreche.., KJ 198\%, S. $186 \mathrm{ff}$. 
linker Ökonomen der Memorandumsgruppe ${ }^{6}$ davon aus, daß vorrangig über eine keynsianische Beschäfrigungs- und Strukrurpolicik die Grundursache der Krise des Sozialstaats, die Árbeitslosigkeir, zu bekämpfen sci. Für das Sozialleistungssystem wird einerseits eine Erhöhung der Einnahmen gefordert durch die Erweicerung des Kreises der Beitragszahler und dic Erhöhung des Einkommensanteils, der zur Beitragszahlung herangezogen wird (Heraufseczung der Beitragsbemessungsgrenze), durch Stcigerung des Staatszuschusses sowie durch eine Umgestaltung der Arbeitgeberbeiträge (sog. Maschinenabgabe und besondere Fonds). Andererseits sollen die Ausgaben rationeller und ursachenbezogener eingesetzc werden, indem die Prävention der Risiken (Krankheit, Arbeirslosigkeit, Unfälle) ausgebaut wird, in der Produktion sozialer Dienstleistungen die privaten Leistungsanbieter (nsozial-industrieller Komplexw) kontrolliert und zugunsten eines öffentlichen, dezcntralen Versorgungssystems zurückgedrängt werden und indem schließlich Einkommen zur Suärkung der Massenkaufkraft und zum sozialen Ausgleich noch gezieiter umverteilt werden (Erhöhung des Kindergeldes, Mindesurente, Abbau der Steuerprivilegien der Besserverdienenden). Auch soll die Selbsthilfebewegung vom Staat und den Sozialleistungsträgern mehr unterstützt werden.

An diesen letzten Punkt knüpft insbesondere eine slinkssozialistisch-grüne" Variantey sozialpolitischer Reformvorschläge an. Sie betont vor allcm, daß die ökonomische und politische Entwicklung seit der Krise 1974 und in Zukunte die Spalcung der Gesellschaft in Arbeitsplazzbesitzer und -nichebesizzer und innerhalb der Arbeitsplatzbesitzer in Stamm- und Randbelegschaften verstärke. Die verstaatlichten und bürokratisierten Sozialleistungsagenturen unterstützten diese Spaltung noch und verhinderten in der Bevölkerung dic autonome Erkenntnis und Befriedigung der eigenen sozialen Bedürfnisse. Perspekrivisch wird ein Ausbau der Basissicherung für alle und die Stärkung der Selbsthilfebewegung in alternativen Formen der Produktion von Sozialleistungen und der Erwerbstätigkeit gefordert.

Nachdem die SPD/FDP-Regierung ihre nur zögernde keynsianische Krisensteucrungspolitik bald nach 1978 aufgegeben hatte, folgten SPD/FDP/CDU grundsätzlich stark dem konscrvativen Lösungsmodell, die Krise durch den forcierten Abbau der Sozialleistungen zu bewältigen. So führten SPD/FDP bis 198 I Kürzungsformen ein, die ideologisch-politisch an sich zum markewirtschaftlich-konservaciven Konzept gehören, wie dic verstärkte Selbstbereiligung in der Krankenversicherung und den Ausbau der bedürfnis- und familienbezogenen Leistungen in der Sozialversicherung. Diese Regierung begründete die starken Kürzungen 1981 ausdrücklich damit, $\mathrm{da} ß \leadsto$ Mißbrauchsmöglichkeiten wirksamer bekämpfr und die Anforderungen an die Selbstverantwortung erhöhe werden ${ }^{3}$. So war es auch nur konsequent, daß die nese CDU/FDP-Regierung 1982 die Vorarbeiten der vorberigen SPD/FDP-Regierung fast lückenlos übernahm. Allerdings machen die Strcichung des Schüler-BAföG 1982/83 und die Kürzungen des Arbeitslosengeldes 1983 den Unterschied zwischen CDU und SPD deutlich: Die CDU geht erheblich radikaler in der Reprivatisierung sozialer Lasten und Risiken vor als die SPD, die sehr viel stärker Elemente des sozialen Ausgleichs und ciner »gercehten Lastenvertcilung « betonc. Mit der sozialpolitischen Programmatik von Albreche und George einerseits sowie den Vorarbeiten in der SPD-Opposicion zu einem umfassenderen sozialpolitischen Programm

6 Vorschläge des DGB zur Wiederhersccllung der Vollbeschäftigung, 1977: Sozialpolitisches Programm dcs DGB yon 19So; 1GM: Soziale Solidaritä, 1977/78; Memonundum 2982, S. 19 Iff; Mcmorandum 1983 , S. $106 f f ., 167$ lf

7 Opiclka, Huber, Wende, Schmollinger, Bucb, Diemer in: Dic Zukunfe des Sozidscaacs (Hrsg. Dic Grïnen Baden-Wurucmberg), 1. Aurl. 1984 ; Strasser, Grenzen des Sozialstaats, 1979.

8 BT-Drucks. $9 / 8,2$, S. 1. 
mit einem Ausbau der Basissicherung sowie der Einnalımen? andererseits scheinen sich zumindest in der Programmatik die alternativen sozialpolitischen Ansätze zrvischen CDU und SPD wieder klarer zu entwickein.

\section{Hauptansatzpunkte und Formen der Kürzungen im Sozialleistungssystem}

Wie in der Krise 1929 ff. sind in der Krise 1974 ff. Hauptansatzpunkre der Kürzungen im Sozialleistungsbereich einerseits der Abbau der Arbeitsłosenversicherung (ArbIV) und des Rebabilitationsrechrs, andererseits die radikale Kürzung des Staatszuschusses zur Sozialversicherung und damit die Abtrennung des Sozialversicherungssystems vom Staatshaushalc ${ }^{10}$.

\subsection{Abbau der staatlichen Arbeitsmarkipolitik}

Schon rein äußerlich wird die Schlüsselstellung der Arbeitsmarktpolitik im Abbau des Sozialleistungssystem dadurch deutlich, daß dieser Bereich als erster im Dezember 1975 erheblich reduziert worden ist. Das Risiko der Arbeirslosigkeit läße sich versicherungsmäßig nicht absichern". Bei wachsender Massenarbcitslosigkeit steigen der Ausfall von Beiträgen wic auch die Ausgaben der ArbJV in unvorhersehbarem Maße, was zwangsläufig die traditionelle Ausfallbürgschafr des Staates in der Arbeitslosenversicherung akrivieren muß (Arr. 120 GG, \$187 AFG). Wäbrend die keynsianische Globalsteuerungspolitik hier zu Anfang noch Auffangpositionen zur Absicherung der Massenkaufkrafi und zur Steverung des Strukturwandels schuf, führe ein zweiter Grund bald zur vollständigen Demontage der Arbeitsmarktpolitik des AFG. Das AFG von 1969 wollte mit der wakriven Arbeitsmarkrpolitik" die Chancen der Arbcitnehmer, insbesondere der "Problemgruppen « auf dem Arbeitsmarkt erhöhen und das Arbeitskräficangebor an qualitacive Änderungen der Nachfrage der Arbeitgeber anpassen. Bei einem Überangebot an Arbeitskräften in allen Qualifikationen, Branchen und Regionen besteht für eine solche Politik kein Bedarf mehr. Dcshalb wurden die wesentlichen Instrumente der "akriven Arbeitsmarktpolitik *, die Förderung der beruflichen Bildung und der Integration behinderter Arbeitnehmer ab 1981 weitgehend abgebaut. Vor allem aber komme der ArblV in der Auseinanderserzung zwischen Kapital und Arbeit während der Krise eine entscheidende Rolle zu. Als Alrernative zum Lohn bestimm die Höhe des Arbeitslosengeldes auch die Höhe des untersten Lohnsatzes, der auf dem Arbeitsmarkt durchgeseczr werden kann, wie ein hohes Arbeitslosengeld den Zwang der Arbeitslosen, jede auch niedrig entlohnte Beschäftigung anzunehmen, abschwächt und damir den Druck der industriellen Rescrvearmec auf die Beschäftigten und ihre Organisarionen mildert. Diese Konzessionsbereitschaft der Arbcitslosen und damit auch der Beschäfcigten kann auch über die direktc Kontrollfunkrion der Arbeitsmarktverwaltung beeinfluße werden, die vor allem über den Zwang auf die Arbeitslosen ausgeübr wird, „zumutbare Arbeit anzunchmen oder ihren Anspruch auf Arbeitslosenunterstützung zu verlieren

9 Siche Zachen (Anm. s) sowie Programm zur zukunlisgercehecn W'citcrentwicklung der Alterssicherung der SPD, FR 27. 3. 1984 5. $1 / 2$.

10 Adamy/Sreffen (Fn. s) sowie Bosch/Priewc. Perspektiven und Handlungsspiclräume der Arbeitsmarkrpoliuk, WSI-Mitt, 1982, S. s7 ff.

II Allg. z.ur Entwicklung und Grundsirukeur der ArblV: Leibfried. Die Inscicucionalisserung der ArblV in

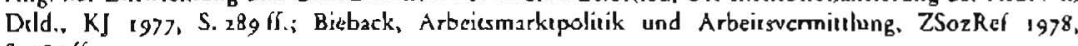
S. 3 s 6 if. 
$(\$ 103,1 \text { I } A F G)^{\prime 2}$. Ähnliche Wirkungen lassen sich über Maßnahmen der beruflichen Bildung und Eingliederung erzielen, an denen teilzunehmen ein Arbeirsloser gezwungen werden kann ( $\$ 119$ AFG), wie über die Schaffung cines "zweiten Arbeitsmarktes durch Arbeitsbeschaffungsmaßnahmen ( $\$ 9 \mathrm{Iff}$. AFG) unter Arbcirsbedingungen, die teilweise unter dem Niveau des übrigen Beschäftigungssystems liegen (Befristung, mangelnde Mitbestimmung und Vertretung).

Seit 1981/82 zichen mehrere Städre auf der Busis der $\$ 18-20,25$ BSHG Sozialhillecmpfänger zu zumutbaren agemeinnütrigen A rbeiten * heran's. In Berlin trägt sich dicses Programm nach amtlichen Angaben selbst's. Bei Arbeit von ${ }^{\prime 2} 0-80$ Stunden in Monat in meist sehr nicdrig qualifizierter Tätigkeic und bei einer Entschädigung von 2-3 DM pro Stunde für vMchrsufwand ( $\$ 19$ Abs. 2 BSHG) geht es um Einubung in Lohnarbcit in ihrer crniedrigsten, perspektivelosesten Form und in offener Konkurrenz zum nfreien Arbeitsmarkt*.

\subsection{Abkoppelung der Sozialversicherung vom Staatshaushalt}

Wegen der ökonomischen und politischen Besonderheiten des Risikos Arbeitslosigkeit ist der Bund zu Zuschüssen für die ArblV und, wegen der besonderen polirischen Belastungen der Rentenversicherung (RV) (z. B. beicragsfrcie Zeiten während des Krieges, Leistungen an Vertriebene etc.), auch für diese zuschußpflichrig (\$1382 ff. RVO). So mußre der Bund der Bundesanstalt für Arbeit (BA) : 975 5,6 Mrd. DM zuweisen und konnte diesen Betrag durch die Kürzungen ab 1976 auf I Mrd. DM 1977 herunterdrücken. Zwar stiegen die Zuweisungen 198 I bis 1983 rviederum aut durchschnitclich i2 Mrd., jedoch konnte ihr weiteres Ansteigen durch Leiscungskürzungen und Beirragssteigerungen verhindert werden"s. Obwohl die "polirischen Leistungen « der Rentenversicherung $z$. Z. noch uber $25 \%$ der Renterausgaben ausmachen, hat der Bund seinen Zuschußantcil von 31\% in 1957 auf $12,9 \%$ 1981 reduziert ${ }^{16}$.

Wesenticher Ansatzpunkr aller Kürzungen in den Sozialleistungsbereichen war es deshalb, den Bundeszuschuß zu den am stärksten kriscnanfälligen Sozialleiscungssystemen der Arbeirslosen- und Rentenversicherung zu kürzen: 1975 Kürzung des Zuschusses zur BA, 1980/81 Kürzung des Zuschusses zur RV um 3,5 Mrd. DM, Y981/82 Kürzung der Defizithaftung an die BA um 9,6 Mrd. DM, Kürzung des Bundeszuschusses an die RV um ca. 1 Mrd. DM, 1982/83 Kürzung des Zuschusses an dic RV um 1, 1 Mrd. DM und der Defizithaftung an die BA um 9 Mrd. DM. Für die Kürzungsrunde $1982 / 83$ sah das folgendermaßen aus (Berräge in Mrd. DM für 1983 , summieren sich in den späteren Jahren auf): [vgl. Graphik auf S. 262]

Der Bund, dessen Steuereinnahmen mit der Krise nur bcgrenzt wachsen, verschafft sich durch diesc Kürzungen im Sozialleistungssystem den Spiclraum für jene politischen und ökonomischen Programme, die höhere Priorität genießen (Förderung der Investitionstätigkeit der Unternehmen, Militärhaushalt ctc.). Diese Entkoppelung der Sozialversicherungshaushale vom Bundeshaushalt führr dazu, daß die Krisenlasten verstärkt von den Mitgliedern der Sozialversicherungssysteme, den Bcitragszahlern und Leiscungsempfängern, d. h. letzelich den abhängig Beschäftigren gerragen werden müssen.

12 Zur hissorischen Entwicklung: Karasch, Der Begriff der Zumulbarkcit in Wandel der Rechesaulfassungen, ZiS 1983, S. 63 ff.

1) Hicrzu: Munder/Burk. Sozialhilic und Arbeitslosigkcir, 1983; Oetker, Anwendung von $\$ 2$ Abs. I BSHG..., DVBI 1983 , S. 1175 .

${ }_{14}$ FAZ 19.4. 1984 , S. 4 .

1s Marcrialienband zum Sozialbudget 1993 , S. 141 . Für 1984 kann sich cvel. sogar ein Úberschuß ergeben: Bosch, Arbeitsmarkepolitik ohne Arbcitslose, Soziale Sicherheit 1984, S. 146 ff.

16 Holfmann, Dic Siabilität des Bundeszuschusses.... DAngVers 1982, S. 401 tf. 


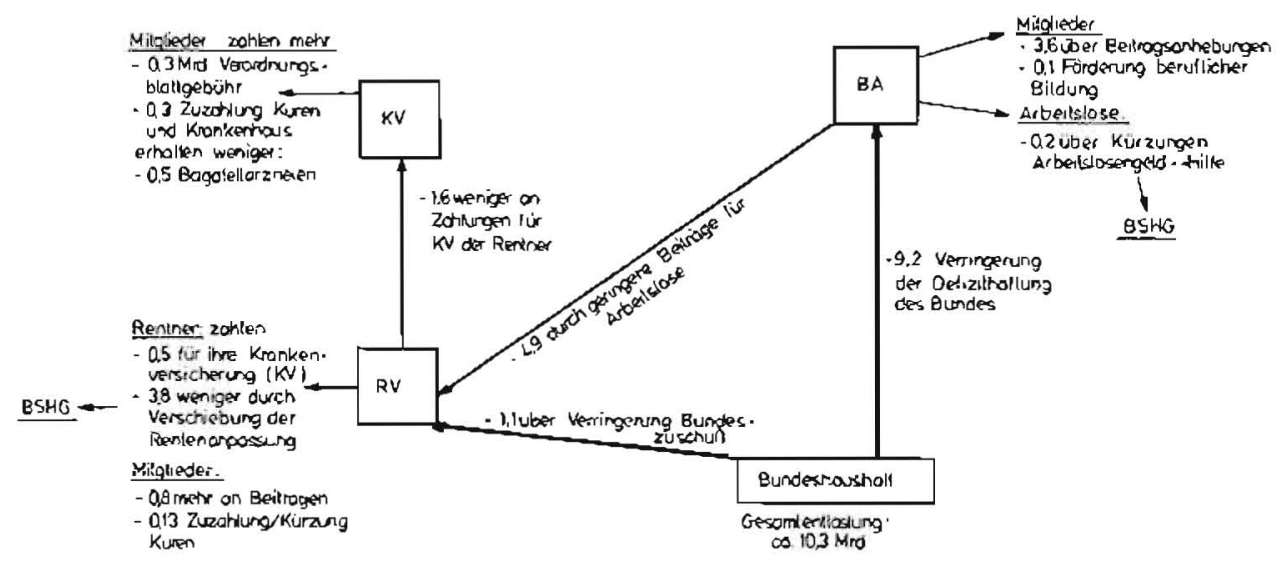

Diese Hauptansaczpunkse der Kürzungen prägen auch ihre verschiedenen Foxmen.

2.3. Die Politik der Verschleierung: Belastung der kleinen öfentichen Haushalte und heimliche Einsparungen

Wie schon dic Graphik zu den Kürzungen $1982 / 83$ zeigt, ist es wohl die wichcigste Form der Kürzunger, die Ausgabenlast bin zu den öffentlichen Etats zu schieben, für die der Bund keine politische Verantwortung trägt und die die Belastung in sehr unterschiedlicher Form, und damit politisch schwer durchschaubar, an ihre Mitglieder weitergeben. Letzelich führen viele Kürzungen dazu, daß die existenzsichernden Basisleistungen (Arbeitslosengeld, Renre) unter den Sozialbilfesatz sinken und damit die Gemeinden über die Sozialhilfe einspringen müssen. Wie diese zusätzliche Belastung der Gemeinden policisch verarbeitet und wcitergegeben wird, ist in jeder Gemeinde unterschiedlich und für den lezztlich betroffenen Bürger meist kaum durchschaubar. Ebenso wurden bevorzuge die Haushalte der RV und der BA auf Kosten der KV saniert, da die über r 200 Krankenkassen diese Belastungen $u$. $a$. in völlig unterschiedlicher Weise an ihre Mitglieder weitergeben müssen (unterschiedliche Beitragserhöhungen, Kürzungen der Ermessensleistungen etc.). So wurde die RV 1977 z. T. dadurch saniert, daß der Beitrag der RV zur KV der Rentner gekürzt wurde; ähnlich verfuhr man 19\$2/83. $1983 / 84$ mußte die KV Beiträge für das von thr gezahlite Krankengeid an dic RV und ArbiV abiühren ( 1 Mrd. DM), die Tbc-Heilmaßnahmen von der RV übcrnehmen $(0,25 \mathrm{Mrd}$. DM) und zugunsten des Bundes die KV der Rentner der Knappschaft tragen $\left(0,8 \mathrm{Mrd}\right.$. DM) ${ }^{18}$.

Zugleich überwiegen mengenmäßig die beimlichen Einsparungen durch Nichtanpassung der bedarfsbezogenen Sozialleistungen an die gestiegenen Lebenshaltungskosten und durch Manipulation der Leiscungsbercchnung. Die größten Einsparungen bei der Sozialhilfe sind dadurch vorgenommen worden', daß die Hilfe zum Lebensunterhalt in den Jahren 1978-83 um ca. $7 \%$ weniger angehoben wurde als in dieser

1) Beträge nach BT-Drucks. $9 / 2074,5.61$ fl, 1 16 ff.

18 Vgl. BT-Drucks, 10/335, S. 64 if.

i) Adumy/Naegele/Stcffen, Sozialstaat odcr Armenhaus, Sozialer Fortschrir ig83, s. igs fi,; Leiblried. FR 21. 10. 1983 , S. 10. 
Zeir die Lebenshaltungskosten für die unteren Einkommensschichten gestiegen waren. Hinzu kommen noch die starken Kürzungen im Haushaltsstrukturgesetz $x 981 / 82$ (vor allem Senkung der Mehrbedarfszuschläge gem. $\$ 23$ BSHG von jo auf $20 \%$ ). 1983 ist die Anpassung zudem um $1 / 2$ Jahr hinausgeschoben worden, wobei nunmehr die Stcigerungstate der Lebenshaleungskosten die Obergrenze für die Anpassung sein soll $(\$ 22$ Abs. 4 BSHG). In ähnlicher Weise sind die Berechrigungsgrenzen für das Wohngeld (Höchstgrenze der unterstützcen Miete) und der Zahlbetrag des Wohngeldes nicht an dic allgemeinen Mietsteigerungen und die Elternfreibeträge und Bedarfssätze des BAföG nicht an die gestiegenen Lebenshaltungskosten und dic entsprechend gestiegenen Löhne angepaßc worden.

In der Sozialhilfe ist zuden die Berechnungsgrundlage für die Hilfe zum Lebensunterhalt, die sog. Warcnkörbe, seit 197 I niche mehr angepaßt worden. Sic sind deshalb veraltec, zu niedrig und in ihrem Verhältnis zucinander überholt (insbes. Energie, persönlicher Bedarf, Emährung etc.). Neue Berechnungen, die eine Steigenung der Regelsäıze der Sozialhilfe um ca. $30 \%$ erbracht hatten, wurden sofort politisch unterd́rückt ${ }^{20}$.

In ähnlicher Weise wurden die Bezugsgrößen der Dynamisierung der RV veränderr, indem ab dem 20. und dem $2 x$. Rentenanpassungsgesetz von 1977 dic Renten nichi wie bis dahin entsprechend den vorher gestiegenen Löhnen, sondern mit erheblich geringeren Steigerungsraten angepaßt wurden. Stant insgesamt um 29,4\% wurden die Renten in dieser Zeit nur um $17.45 \%$ erhöht, wodurch laufend $12 \%$ der Rentenzahlungen eingespart werden ( $1982: 19$ Mrd. DM). Ab r 983 werden die Renten jeweils um $1 / 2$ Jahr später zum 1. 7. erhöht. Für das Jahr I 984 wurde die Anpassung statt aú die durchschnitzliche Steigerungsrate der vorherigen 3 Jahre nur noch aul das letzte Jahr bezogen: Dadurch wurden wiedcrum ca. 1,s Mrd. DM eingespart.

Während die Bciträge zur BA auf der Basis des gesamicn Lohneinkommens berechnet werden ( $\$ 175$ AFG), werden bei der Berechnung des Arbeirslosengeldes (Alg) und der Arbeicslosenhilfe (Alhi) schon traditionell die Überstundenverdienste und -zuschläge nicht berücksichtigt ( $\$$ i 2 Abs. 2 AFG). Das AFKG von 198 1 schloB ab 1982 auch die einmaligen und wiederkehrenden Lohnzahlungen (13. Monatsgehalt, Urlaubsgeld) bei der Bercchnung des Alg aus ( $\$$ i 2 Abs. 2, S. 2 AFG), dic regelmäBig 10\% des Jahreslohns ausmachen. Bei Berufsanfängern wird das Alg statt nach dem vollen nur noch nach der Hälfte des zu erwartenden Arbeitsentgelts bemessen (\$ r 2 Abs. $5 \mathrm{Nr}$. $\mathrm{AFG}$ ).

Durch diese Kürzungen bei den exiscenziell wicheigen Lohnersatzleistungen der Basisversorgung (Alg, Renten) werden das Sozialleistungseinkommen vieler Bevölkerungskreisc unter die Sozialhilfcsät»e, die Sozialhilfesätze wiederum unter das existenziell Nocwendige gedrückt (s. u. 3.t.).

\subsection{Ausgrenzungen und Belasiungserböhungen}

Ausgrenzungen aus dem Kreis der Anspruchsberechigien geschahen offen durch die fast vollständige Beseirigung des Schüler-BAföG ab 1983 und dadurch, daB im AFG seit 1976 bzw. 1981 keine Leiscungen der beruflichen Förderung, der Arbeitsbeschaffungsmaßnahmen und der (originären) Alhi an Personen erbracht werden, die vorher nicht versichert waren. So reduzierte sich durch die Beseitigung der (originären) Alhi an arbeitslose Schul- und Studienabgänger 1982 die Zahl der Empfänger von Alhi auf die Hälftere. 
Verdeckter wirkt diese Kurzungsform, indem dic Anwarschafuszeiten für Versicherungsleistungen erhöht wurden und in einer engen Rahmenfrist vor dern Versicherungsfall erfüllt sein mussen, so für die berufliche und medizinische Rehabilitation in der RV (ab 1982), für dic vorzeitigen Renten wcgen Arbeisslosigkeit (ab 1982) und wegen Beruls- und Erwerbsunfähigkeir (ab 1984) ${ }^{22}$. Für den Bezug des Arbeisslosengeldes waren früher 6 Beschäfrigungsmonate nowwendig, um einen Anspruch auf Arbeisslosengeld fur 3 Monare zu begrïnden; ab 1982 sind es $1 \mathrm{Jahr}$ für $1 / 2 \mathrm{~J}$ ahr Arbcitslosengeld und ab 1983 i Jahr für 4 Monatc Arbeicslosengeld (\$104, 106 AFG). Diese Kürzungsformen grenzen jenc aus dem Soziallcistungssystem aus, die nur eine unregelmoßige, unterbrochenc oder gar keine Erwerbstätigkeit vorweisen können (Frauen, Jugendliche, Gelegenheits-Arbeitnehmer exc.).

Von bedarfsbezogenen Leistungen können dadurch immer mehr Personen ausgeschiossen werden, daß das anzurechnende Einkommen ihrer Unterhakisverpflichteten nicht entsprechend den Preissteigerungs- und Lohnsteigerungsraten erhöht wird. Dies gilt z. B. für die mangelnde Anpassung der Elternfreiberräge beim BAföG und vor allem für die Arbeitslosenhilfe, bei der das anzurechnende Einkommen des Ehegaten seit i 979 ab 75,- DM wöchentlich beginne (+ 35,- DM wöchentlich für jedes Kind, $\$ 13^{8}$ Abs. I Nr. 2 AFG), weshalb verheiratete Frauen und unterhalssberechtigte Minderjährige meist keine Arbeitslosenhilfe mehr erhalten.

Leistungskürzungen der Höbe nach sind im Basissystem des Arbeitslosengeldes erst für das Jahr $19 \$_{4}$ vorgenommen worden, nachdem die Kürzung der Bemcssungsgrundlage weitgehend ausgeschöpft war. Arbeitnehmer ohne Unterhaltspflicht gegenüber Kindern erhalten nunmehr statt $68 \% 63 \%$ und statt $58 \%$ s6\% ihres vorherigen (eh schon gekürzten) Entgelts; ähnliche Sätze galten schon vor 1969 . Die Renten wurden dadurch gekürzt, daß die Rentner ab 1983 bis 1985 allmählich $5 \%$ ihrer Rente und ihres anderen Einkommens für die Krankenversicherung der Rentner abü̈hren müssen ( $\$ 1304 \mathrm{c}$ RVO). Ebenfalls wurde der Kinderzuschuß in der RV ab r 984 durch das erheblich geringere Kindergeld erseczt. In der Krankenversicherung wird das Krankengeld ab 1984 für die Versicherten um $11,5 \%$ gekürzr, da von ihm nunmehr Beirräge zur RV und ArblV zu zahlen sind.

In allen Sozialversicherungsbereichen ist der Lohnersatz wahrend einer Rehabilitationsmaßnahme (Übergangsgeld, Unterhaltsgeld'), der früher dem vorherigen Netroentgelt cntsprach, allmählich auf nunmehr $75 \%$ (Versicherte mir Unterhaitsphiche) bzor. $65 \%$ (Versicherte ohne Untcrhailspflichı) gesenkı worden. Für das Unterhalrsgeld bei allgemeinen Bildungsmaßnahmen rach dem AFG gelten die Sátze von $70 \%$ bzw. 63\% lür vorher Arbeitslose bzw. von Arbeitslosigkeit Bedrohte, ansonscen wird nur noch ein Darlehen nach Ernessen in Höhe von $\{8 \%$ des vorherigen Nettoentgelts gcrvährn.

Furr $19 \$ 4$ ist dic Lcistung während des Mutterschalisurlaub von vorher 750,- DM auf \&10,-DM monalich gekürzl ( $\$ 200 \mathrm{Abs}, 4$ RVO), wie das Kindergeld $\mathrm{r}^{8} \mathrm{~g}_{2}$ aligemein und für $1983 \mathrm{ab}$ einer bestimmen Einkommensgrenze reduzien wurde. Die Sozialhilfe wurde durch den Abbau der Mehrbed arfszuschläge, in der Blindenhilfe und in der Überbrückungshilfe gekürzt, wie sie bei Asylbewerbern nach dem Ermessen der Verwaltung auf Naturalleistungen und ein Minimum begrenzı werden kann.

Früher hatce die RV die Risiken der krankheitsbedingten Arbeitsunfähigkeir und der Arbcitslosigkeit insoweit mitgetragen, als dicse beitragslosen Zeiten bei der Rentenberechnung wie Beitragszeiren berücksichrigt wurden (sog. Ausfallzeiten). Die Notwendigkeit, die RV finanziell zu enclasten, führe dazu, diese sog. sekundären Risiken fehlender Beitragszahlung in der RV durch jene Sozialleistungssysteme abzusichern, die auch das primärc Risiko abdecken. So mußtc die BA ab 1978 Beiträgc an die RV für die Zeit der Arbeicslosigkeit ihrer Leistungsbezieher abführen, wobei

22 Dies wurde nicht dadurch wetigemachi, daß die Anwartschaft fur die Alrersrente von is auf s Jahre Bcitragszeiten reduziert wurde, da die Altersrente erst ab dem 65 . Lebensjahr, dic gleichhohe Eracrbsunfähigkeitsrentc aber olne Altersbegtenzung bexogen werden konntc. Dir Nettosparcifekt bercchnetc sich It. BT-Drucks. 10/335. S. 52, von $1984-1987$ auf 2.7 Mrd. DM. 
diese Beiträge ab 1983 nur noch auf der Basis des Alg bzw. der Alhi berechnet werden und Arbeitslosigkeit wieder zur Ausfallzeit wurde ( $\$ 1259$ Abs. s Nr. 3, 3a $\mathrm{RVO}$ ). Ab $19 \$_{4}$ müssen aus dem Krankengeld der KV und dem Verletzien- und Übergangsgeld der UV Beiträge an die RV abgeführt werden ( $\$ 3_{3}^{8} 5 \mathrm{~b}$ RVO). Diese an sich sinnvolle Neuverteilung der Risiken führte jedoch zugleich bei den Versicherten zu ciner Kürzung dieser eh' nur den Nerto-Lohn ersetzenden Leistungen um die Beiträge zur RV.

Die Selbstbeteiligung, die es in der KV in wechseinden Formen schon beim Bezug von Arznei-, Verbands-, Heil- und Hilfsmitteln sowie Fahrtkosten gab, ist für r 982 erheblich erhöht und für 1983 bei einem Krankenhausaufenthalt pro Tag auf 5,- DM und bei einem Kuraufenthalt pro Tag auf $10,-D M$ in allen Zweigen der Sozialversicherung neu eingeführt worden. Die Verordnung von sog. Bagatell-Arzncimitteln muß dcr Versicherte nunmehr allein tragen. Von dicsen Vorschriften gibe es wiederum Ausnahmen bei Unzumurbarkeit für den Versicherten. Beitragserböhungen fanden auch schon von r950-1974 statc (RV von $10 \%$ auf $18 \%, \mathrm{KV}$ von durchschnitrlich $5,2 \%$ auf $9,5 \%$ ), konnten jedoch durch dic allgemein steigenden Reallöhne aufgefangen werden. Dagegen mußten sich Beitragserhöhungen in der ArblV von 1982 von $3 \%$ auf $4 \%$ und für 1983 auf $4,6 \%$ und in der RV für 1983 von $18 \%$ auf $\times 8,5 \%$ erheblich auswirken, da die Reallöhne ab 198 i sanken"s. 4,5 Mrd. DM Mehreinnahmen werden für 1984 allein daraus erwarter, daß die einmaligen Sonderzahlungen (Weihnaches- und Urlaubsgeld) nun nicht mehr nur bis zur Beitragsbemessungsgrenze des Auszahlungsmonats, sondern in voller Höhe verteilt auf das ganze Jahr bei der Beitragszahlung zu berücksichrigen sind.

\subsection{Ausbau des Kontrollapparats und Einsparungen im Verwaltungsvollzug}

Die Kontrollen des Leistungsbezugs sind vor allem im AFG gem. $\$ 103$ und 119 AFG und $\$ 25$ BSHG bei den Anforderungen an die Leistungsbezieher, zumutbare Arbeit anzunehmen, in wechselnden Formen und wechselndem Ausmaß erheblich erhöhe worden ${ }^{23}$. Ebenfalls wurde die Dauer der Sperrzeiten, die bei ersmaliger Verweigerung einer zumurbaren Arbeit oder bei verschuldeter Herbeiführung der Arbcicslosigkeir verhängt werden können, von früher 2 Wochen 1978 auf 4 und 1981 auf 8 Wochen erhöhc. Scieg die Zahl der Sperrzeiten zu Beginn der Krisc stark an"s, so ist sie trotz Verschärfung im Jahr 1982 zurückgegangen, da die BA den Arbeitslosen kaum noch offene Stellen anbieten kann. In Berlin hat die konsequente Durchsetzung des Zwangs zu nzumurbarer, gemeinnürziger Arbeic a (\$2 5 BSHG) dazu geführt, daß 1983 zum ersten Mal seit 1970 die Zahl der Empfänger von laufender Hilfe zum Lebensunterhal rückäufig is ${ }^{26}$.

Eher symbolisch-ideologische Einsparungen kann man die vollständige Umstellung des Studenten-BAföG auf Darlehen ab 1983 nennen, da sie zu kciner akruellen Ausgabenenclastung führen, das Darlehen unverzinst und mit sehr langen Rücklaufzeiten versehen ist und dadurch für den Fiskus in seinem Wert erheblich gemindert wird und der Verwaltungsaufwand sehr hoch ist. Das Ziel, über einen sozialen Numerus Clausus die ch zu hohe Zahl der Studenten wieder zu senken und stärker

23 Übersiche über dic Soxiale Sicherheil (Hrsg. BMLA), 9977, S. 109 und Materialienband zum Sozialbudgee 1983, S. 240.

i4 Vgl. oben Fn. 1 1, 13.

as Bicback, Statusschurz und Mobilitätszwang ..., DuR 1977, S. s $16 ., 19 / 20$ sowie Geschältsbericht der BA

$1982,1983,5.24$.

$26 \mathrm{Vgl}$ aben Fn. 14. 
ökonomische Hebel in die Studienpianung und den Srudienablauf einzubauen, ist deshalb überdeuclich.

Groß sind die Einsparungen im Verwaltungsvollzug. Als Beispiel sei hier die Verwaltungspraxis der Sozialhilfe genannt. Trotz allgemeiner bundesweiter gesetzlicher Regelung gibt es erhebliche regionale Unterschiede des durchschnitclichen ProKopf-Aufwandes an Sozialhilfe ("Schattenregelsätze* mir leichtem Nord-SüdGefälle)" ${ }^{27}$. Die Gemcinden versuchen gerade jene zahlreichen Leistungen, die in ihrem Ermessen stehen, wie z. B. die einmaligen Hilfen zum Lebensunterhalt (Weihnaches-, Heizungs- und Kleidungsbeihilfe) erheblich zu kürzen ${ }^{28}$. Weiterhin zählt hierzu dic bewußt restriktive und abweisende Ausgestaltung von Verwaltungsverfahren und Organisation, wodurch die schon traditionell bestehenden hohen Zugangsbarrieren noch verstärkt werden ${ }^{19}$. Ein weiteres Bcispiel ist die Ausgestaltung der Arbeitsvermittlung der BA. Da der Eriolg der Arbeitsvermitcler institutionsintern an der Quantitä der Vermirtlungen gemessen wird, konzentriert sich die Vermirtlungstärigkeit auf die Wünsche der Arbeitgeber und auf die leichter vermittelbaren Arbeicslosen ${ }^{30}$ und klassifiziert $x$. T. die Arbeitslosen in leichr-, schwer- und gar nich vermittelbarc ${ }^{31}$. Zudem werden längerfristig arbeisslose Ausländer für nich mehr vermittelbar erklärt, weshalb ihnen alle Leistungen nach dem AFG enczogen werden und sie Sozialhilfe bezichen müssen, so daß ibnen die Ausweisung drohs $(\$ 10 \text { Abs. } 1 \text { Nr. } 10 \text { Aus IG) })^{32}$.

Gerade diesc Veränderungen im Verwaltungsvollzug becreffen jene Problemgruppen des Arbeitsmarkecs und der Bevölkerung, die weder gesarntgesellschaftlich noch in ihrem einzelncn Auftreten sozialc und politische Durchsetzungschancen und Kompetenzen haben: Ausländer, Frauen, Jugendliche, An- und Ungelernte. Dadurch haben dic Kürzungen und ihre verwaltungsmäßige Umsetzung nur jene Mechanismen noch verstärkt, die auch zu Zeiten der guten Konjunktur geltcn: So har die Gewährung fördernder Sozialleistungen nach dem AFG" wie der Basissicherung der Sozialhilfe ${ }^{34}$ die relativen sozialen Unterschiede, dic unter den Adressaten dieser Leistungen bestehen, noch verstärkt, statt sie zugunsten der besonders sozial Benachteiligten zu beseitigen.

Die Kürzungen der „Realtransfers $\alpha$, zu denen alle staatlichen/öffentlichen Dienstleistungen zählen, können auch nich annäherungsweise zusammenhängend erfaßs und bewertet werden. Beispielhaft seien nur genannt die Kürzungen im Sozialisationsbereich (Kindergärten, Jugendhilfe), im Bildungsbereich (Schulen, Büchereien), Kulturbereich (Theater etc.) und im Infrastrukturbercich (Nahverkehrsmitcel, öffentliche Einrichtungen wie Schwimmbäder etc.). Von ihnen werden insbesondere wieder jene Bevölkerungsschichten betroffen, die sich diese Dienstleistungen niche privat auf dem Markt ersaczweise beschaffen können, sondern auf ihre staacliche/öffentliche Bereitstellung angewiesen sind's.

Ansätze verstärkter Öffentlicbleeit sowic Kontroll- und Koordinationsinstrumente

27 Leibfried, Zur Socialpolitik der Vercilungsformen in der Sozialhille, NDV s\$8 1 , S. 26t ff.

$28 \mathrm{Vgl}$. Bürgerschafisdrucks. HH 10/458, S. 3, 26 if.

29 Vol. Leibfried, Armutspotential und Sozialhille in der Bundesrepublik, KJ 1976, S. 377 fl.; Hanmann. Soziallhilfebedürfúgkeit und .Dunkelziffer der Armut $4,198 \mathrm{~s}$.

30 Pollmeicr, Zur sozialen Ausgestaltung der Arberitsvermierdung. Soziale Sicherheil 1982, S. 273 ff.

31 TAZ (Hamburg), 8.2.19\$

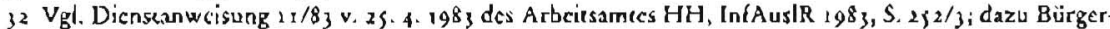
schafusdrucks. $\mathrm{HH} \mathrm{I} 1 / 1097$ und 1187 .

13 Förderung der berufichen Bildung (Hrsg. BA), 1975, S. 16 fl; Pintar, Die Enıscheidung zur Unschulung, 1978, S. 32 ff.

34 Leiblricd, Fn. 29.

is $\mathrm{Zu}$ Umfang und zur Unverceilungrwirkung der Rtaleransfers: Transfer-Enquéte-Kommission, Das Trunsiersystem in der Bundestepublik Deld., 1981, S. 62 f. 
zwischen den Verbänden der Leistungsersteller und Leiscungserbringer (konzerviene Akrion, vertragliche Begrenzung der Verschreibung von Heil- und Arzncimittel durch die Ärzte, Vertragssysteme zwischen Krankenhäuscm und Krankenkassen) sollen die starken Kostensteigerungen, die auf den nicht funktionsfähigen Markt für Gesundheitsleistungen zurückzuführen sind, eindämmen. Jedoch hat diese Politik der "Seelenmassagea ("moral persuasiona) nur begrenzten Eriolg gebabt.

3. Auswirkungen auf das System der Sozialleistungen: weder Statusschutz noch Bedarfsdeckung

\subsection{Bedarfsbezogene Leistungen werden vom Bedarf abgekoppelt}

Besonders schwerwiegend ist der Systembruch in der Sozialhilfe. Der Regelsatz ist wie gezeigt - politisch weit unter das Maß der "Menschenwürdew (\$S I und 12 BSHG) gedrückt worden ${ }^{36}$. Da der Abbau des Reallohneinkommens ab 1981 und des Sozialeinkommens ab 1976 zunehmend mehr Tcile der Bevölkerung auf das Sozialhilfcniveau sinken läßc, sind vor allem die Armen der Armen von diesen Kürzungen betroffen. Die Basis des Sozialleistungssystems in der Bundesrepublik, die Sicherung des Existenzminimums durch die Sozialhilfe, ist damit zerbrochen.

\subsection{Reprivatisierung der Familienlasten}

Dic erheblichen Kürzungen des BAföG, insbesondere des Schüler-BAföG, haben die Ausbildungsfunktion weitgehend wieder in die Familie zurückverlagert und reprivatisicrt. Ebenso wirkten die Kürzungen des Kindergeldes 1982 und 1983 und des Muteerschaftsgeldes 1984. Abgesclilossen wird diese Politik durch die geplante Sreuerreform, durch die den Familien noch nicht einmal wiedergegeben wird, was itmen vorher insgesamt genommen worden ist, und die zudem als Familienlastenausgleich nur das individuell varfügbare Einkommen durch Steuervergünstigungen erhöhen wilk, nicht aber die allgemeinen sozialen, umverteilenden Instrumente des Familienlastenausgleichs stärkt (Kindergcld, Familienbezug der Ausbildungsscipendien und des Wohngeldcs, Babyjahre in der RV, Realtransfers durch Kindergärten etc.). Auch die Differenzierung der Lohnersatzleistungen der Sozialversicherung (SV) während Rehabilitationsmaßnahmen nach den Unterhaltsverpflichtungen gegenüber der Familic isı eher eine soziale Verkleidung der rigorosen Kürzungen in diesem Bereich ais eine familienpolitische Wohltat.

\subsection{Zerstörung des Konzepts der saktiven Arbeitsmarktpolitik" und der Sicherungsfunkion der Arbeitslosenversicherung}

Durch die rigiden Kürzungen der Lohnersatzleistungen bei den allgemeinen $\mathrm{Maß}$ nahmen der beruffichen Bildung und bej der Rehabilitation Behinderter sowie der Förderung der Mobilitäc der Arbeitnehmer und der Schaffung von Arbeitsplärzen sind die Leiscungen des AFG ab 1976, besonders seit 1981/82 an wieder rein reaktiv auf die Beseitigung von Arbeitslosigkeit begrenzt. An die Stelle positiver finanzieller Anreize ist die Drohung, das Arbeitslosengeld zu entziehen, getrezen, falls der 
Arbeitslose nicht bereit ist, sich den veränderten Bedingungen des Arbeitsmarktes anzupassen und notwendige Bildungsmaßnahmen über sich ergehen zu lassen ( 85103,119 AFG). Damit ist das - vom AFG 1969 verfolgte - Grundkonzept der aktiven Arbeitsmarktpolitik zerstört werden ${ }^{3}$.

Zudem verliert die ArblV immer melır ihre eigenständige Sicherungsfunkuion, indem Versicherte in die Sozialhilfe abgedrängt werden. Zu den schon erwähnten Kürzungen in den Voraussetzungen (Anwartschaftszeiren, Berechnung und Höhc des Alg und der Alhi, Nichtanpassung der Freibeträge für das anzurechnende Einkommen des Ehepartners bei der Alhi) kommt noch hinzu, daß die Alhi eines Arbeitslosen, der sein früheres Arbeirsentgelt nicht mehr erreichen kann - was bei Qualifikationsverlust durch längere Arbeitslosigkeit meist der Fall ist - gem. \$136 Abs. 2, 112 Abs. 7. AFG neu und geringer festgesetzı werden kann. Die Konsequenz dieser Maßnahme ist, daß immer mebr Arbeirslose keine oder nur so geringe Leistungen der BA erhalten, so daß sie auf Sozialhilfe angewiesen sind ${ }^{38}$. So erhielten im April 1984 nur noch $39 \%$ aller registrierten Arbeisslosen Alg und 27,1\% Alhj'9. Berücksichrigt man, daß zu der offiziell ausgerviesenen und registrierten Arbeisslosigkcir mindestens noch einmal 50\% nicht registrierter, sog. verdeckter Arbeitslosigkeit hinzukommt, so erhielten 1982 ca. 1,3 Mio. Erwerbslose keine Arbeitslosenunterstützung; diese Zahl dürfte sich 1983 auf r,6-2 Mio. erhöhr haben ${ }^{40}$. Auch zeigt die starke Konjunkturabbängigkeit der Beantragung vorzeitiger Renten der RV (Berufsund Erwerbsunfähigkeitsrenten gem. I $\left(246 / 47\right.$ RVO ${ }^{4}$, daß vielc Arbeitslose in diese Renten ausweichen, um ibre Einkommenssituation zumindest zu stabilisieren.

Damit haben die Arbeitsfördenung und die Arbeitslosenversicherung ilhre Schurzfunktion in der Krise weitgehend verloren, dient ilhre Destruktion gerade dazu, wie oben gezeigr, den Druck auf die noch Beschäfrigten zu erhöhen und dic Krisenlasten auf die Arbeitslosen abzuwälzen. Verfassungsrechtich ist cs kaum noch zu rechtefertigen, die Arbeitnehmer in ein Sondersystem zu zwingen und ihnen dafür Beiträge abzuverlangen, wenn dieses Sondersystem für viele von ihnen doch nur Leistungen gewährt, die unterhalb der Sozialhilfe liegen und die durch jenes allgemeine System der Sozialhilfe aufgestockt werden müssen, das jeder Bürger ohne vorherige Beitragszahiung beanspruchen kann.

\section{4. Änderung der Leistungsprinzipien der Sozialversicherung}

Bisher waren Alg, Alhi und alle anderen Lohnersatzleistungen der SV gemäß dem Prinzip der individuellen Äquivalenz ${ }^{42}$, d. h. nach der Höhe des vorherigen individuellen Beirrags und Lohns, berechnet worden. Dagegen sind die Sachleiscungen der Sozialversicherung (z.B. Krankenpflege, Dienstleistungen, sächliche Mirtel der Rehabilitation etc.) traditionell nach Bedarfsgesichtspunkten ausgerichtet. Dieses

\footnotetext{
37 Bosch/Pricwe, Fn. 10.

38 Nach ciner Untersuchung der Hans-Bockler-Suifung, Die néue Armut, Ausgrenzung von Arbeitslosen aus dec Arbeicslosenunterstützung, 1983 , S. 47 f. crhicleco im September r 982 c2 $46 \%$ der länger als । Jahr registrierten Arbeieslosen keine Leistungen nach dem AFG, sowie nach cince Umfrage im September 1983 in Dormund und Essen 33,3 bzw. $18.8 \%$ aller registrieren Arbeitslosen Soyialhilfeempännger waren (cbenda, S. 107 If.) und nath Angaben des Deutschen Städrctages 1989/4 der Antcil der Arbeitslosen an der Gesamizahl der Emplänger von Hilfe zum Lebensuntcrhale auf $25 \%$, in manchen Zeneren der Arbeislosigkeit g1x auf $35-40 \%$ gesticgen sei (TR v. 14.4. 1984, S. r).

39) BT-Drucks. $10 / 145$ i v. 18,1984, S. 7 .

40 Hans-Böckler-Stifung cbenda, S. 76 If, dort auch zur Problemacik der olíziellen Arbeitslosensuatisuik. Zur verwaltungsmäßigen Schionung der registrierten Arbeitslosenzahlen vgl. FR 11. 10. 83 , S. 4

${ }_{4} 1$ Kolb, Zur Finanzicrung der geserzlichen RV .... DRV $1983,5.8$ iff., $93 / 4$.

42 Vgl. oben Fn. 11 .
} 
System, das einerseits den relariven Lebensstandard und Status über die nach dem vorherigen Lohn berechneten Lohnersatzleistungen sichert, andererseits wichtige Dienstleistungen allen Versicherten unabhängig von der Höhe des vorherigen Beitrags zugänglich macht, ist seit der Krise 1974 stark durchlöchert worden.

\subsubsection{Einschränkung der Versicherungsäquivalenz bei den Lobnersalzleistungen der Sozialversicherung}

Die individuelle, versicherungsmäßigc Äquivalenz bei den Lohnersarzleistungen der SV ist zucrst im AFG eingeschränkt worden, indem zwar der gesamte Lohn bis zur Beirragsbemessungsgrenze für dje Beirragszahlung herangezogen wird, bei der Leistungsbemessung jedoch wesentliche Lohnbestandteile fortallen: Schon traditionell die Überstundenvergüıungen und nunmehr seit 1982 auch dic einmaligen und wiederkehrenden Lohnzahlungen. Dicse Diskrepanz hat auch die anderen Sozialversicherungszweige erfaße. Seit $198_{4}$ werden die wiederkchrenden einmal jährlichen Sonderzahlungen anceilig auf das ganze Jahr verteilt und dadurch stärker zur Bcitragszahlung herangezogen; bei der Leistungsgewährung aber werden sie nur dann berücksichtigt, wenn sie im sogenannten Bemessungszeitraum 3 oder 4 Wochen vor Eintritt des Versicherungsfalls ausgezahlt worden sínd ${ }^{43}$. Schließlich wurde die Steigerung der Renten 1977-\$1 yom Wachstum der Löhne und Beiträge abgekoppelt. Zwar hat der Geserzgeber Spielraum, die Leistungsgervährung von der Beitragsberechnung und -zahlung abzukoppeln ${ }^{44}$. Jedoch verliert das Sozialversicherungssystem seine institutionelle und verfassungspolitische Basis, wenn der Beitrag immer mehr dazu dient, ein staatliches Leistungssystem nur in allgemeiner Weise wic eine Steuer zu finanzierents.

\subsubsection{Familien- und Bedarfsbezug der Lohnersatzleistungen}

Auch hier hat dic ArblV eine Vorreiterrolle gespiclt. Wegen der historischen und sozialpolitischen Nähe der Unterstützungsleistungen der Arbeitslosenversicherung (insbes. Alhi) zur Sozialhilfe hatce der Vorläufer des AFG, das AVAVG, das Alg durch Zuschläge je nach Familienstand den besonderen Bedarfslagen angepaßt. In geringem Maße gilt dies auch für die Renten der RV ${ }^{46}$. Hatte das AFG diesen Familienbezug der Lohnersatzleistungen abgeschaffr, so wurde er ab 1976 dadurch wieder cingeführt, daß das dem Alg zugrundeliegende Netroarbeitsentgelr nach Steuertabellensätzen zu berechnen ist, dic den Familienstand mic berücksichtigen (\$ I I AFG). Sodann wurden die Lohnersazzleiscungen während der allgemeinen Bildungsmaßnalumen des AFG und während der Rehabilitationsmaßnahmen in allen Zweigen der $S V$ in der Höhe um $10 \%$ danach differenziert, ob der Versicherte Unterhaltspflichcen hat oder nichr. Ab 1984 hat diese Differenzicrung auch dic Basisleistung des $\mathrm{Alg}$ und der Alhi $(68 \%$ zu $63 \%$, $58 \%$ zu $56 \%)$ erfaßt.

\subsubsection{Umbas des Rebabilitationskonzepts der Sozialversicherung}

Wahrend die durch das Rehabilitationsangleichungsgesetz von 1974 ausgebauren sachlichen Leistungen der medizinıschen und beruflichen Rehabilıtation Bchunderter

43 BSGE \{3, S. 122 ff. m.w.N.

44 So zur Nichtberucksichtigung von Überstundenvergutungen beim Arblg: BVerGE \{1, IIs ff.

45 Zu den sozialpolitischen und rechelichen Grundlagen der Beirragsfinamzierung: Zacher (Hrsg.). Die Rolle des Beirrags in der sozialen Sicherung, 1980.

46 Der Kindercuschuß $(\$ 1262 \mathrm{RVO})$ ist ab $198_{4}$ für Neureneen abgeschalf und ganr auf das Kindergeld reduziert worden. Familienbezogen sind auch die Hinterblicbenenrenten gem. $\$ \$ 1263$ ff. RVO. 
(Therapie, Berufsausbildung etc.) durchweg erhalten geblicben sind, wurden die Lohnersatzleistungen, die an Versicherte ährend der Dauer einer Rehabilitationsmaßnahme gezahle werden, in allen Zweigen der Sozialversicherung erheblich, in der beruflichen Rehabilitation bis auf den Satz des Arbeirslosengeldes gekürzt. Die oft persönlich sehr belastenden Rehabilitationsmaßnahmen werden so ohnc jeden finanziellen Anreiz angeboren und verlangen nunmehr erhebliche finanzielle Opfer. Die scharfe Konkurrenz auf dem Arbeitsmarkt wird die vorzeirig behindcrien Arbeitnehmer zwingen, ihr A rbeirsvermögen ihren veränderten Fähigkeiten und den neuen Marktbedingungen anzupassen, wobei dieser indirekı Zwang noch durch den direkten Zrvang, bei Verweigerung einer Rehabilitationsmaßnahme die Sozialleiscungsansprüche zu kürzen $(\$ \$ 66,68$ SGB AT, 19 Abs. 1 Nr. 3 AFG) verschärft wird ${ }^{4}$. Auch der präventive Charakter der Rehabilitacion ist durch die erhöhten Anwartschaften bei der Leistung von Kuren und die Verlängerung der Intervalldauer zwischen zwei Kuren ( $\$ 236$ RVO) reduziert worden ${ }^{4}$.

\subsubsection{Beschränkung der Bedarfsoriencierung bei den Sacbleistungen}

Während die Lohnersatzleistungen zunehmend nach Bedarfsgesichtspunkten vom vorherigen Lohn und Beitrag abgekoppcle werden, werden umgekehrr die bedarfsbezogenen Sachleisiungen der Sozialversichcrung durch die gestiegcnen Selbstbereiligungsquoren stärker an die finanzielle Leistungsfähigkeit des Versicherten gebunden. Andererseics wird die Selbstbeteiligung über Ausnahme- und Härteregelungen ( $\$ 182 a, 182 f, 182 c, 184,184 a, 1243$ RVO) in der Praxis zumindest dann ausgeselzr, wenn das Einkommen des Versicherren nicht weit über dem Sozialhilfeniveau liegt. Die Spareffizienz dieser Kürzungen ist aufgrund des hohen Verwaltungsaufwands bcim Einzug der Selbstbeteiligungsgelder wie bei der Venwaltung der Ausnahmeregelungen sehr zweifelhaft, niche dagegen die sozialpolitische Auswirkung, einkommensschwache Versicherte von der Leistungsinanspruchnahme auszuschließen ${ }^{49}$.

\subsection{Abbau der Rechtssicherbeit - Relativierung des allgemeinen Leistungsgesetzes}

In den existenziellen Bereichen der sozialen Sicherung hat das allgemeine Leistungsgeserz auch die Funkrion, dem Bürger Rechtssicherheit zu geben, d. h. ihm eine längerfristige Lebensplanung zu ermöglichen, die Leistungsgewährung vorher abstrakr geserzlich fesczulegen und damir die Bürger unablängiger von der *Willküru der Leistungsbürokratie zu machen. Beide Funktionen hat die Krise 1974 ff. zerstört. Einmal wurden die ökonomischen Krisenfolgen und die Resultate der ökonomischen und politischen Verteilungskämple sofort in Maßnahmegesetze umgeserze, die den Standard der sozialen Sicherheit der abhängig Beschäftigren jeweils situativ neu festlegten und damir die Verläßlichkeit des Systems weitgehend zerstörten. Auch hat das Übergangsrecht die Kürzungen schon auf laufende Leistungsverhältnisse angewandr ${ }^{\circ}$. Zum anderen wurden, wie gezeigt, selbst die Leistungen in der klassischen Sozialversicherung zunehmend von Bedartsgesichtspunkten abhän-

47 Vg) Bicback, ober, Fn, 25, S. $5(f,, 181$.

$4^{8}$ Dic Kuren sind um meht als 1/2 zuruckgegangen: FAZ v. 9. \$. 1983 sowic Rentenanpassungsberichee $9^{9} 2$ und 1983, BT. Drucks. $9 / 1551$ S. $23,10 / 560,5.24$

49 Vyl. Heizzcr. Der Härcilall - Ein Schlupiloch zur Systcmveränderung, Soziale Sicherheit II/19\$z: Breucr, Ein Spargeselx, mil Mehrkosicneffekt, Dic Sorialversicherung r $98_{3}$, S. 7 ff. Zur Fesclegung der - Härex und zur Bedeutung des Sozialhilfeniveaus BSGE $\{2,267 \mathrm{fl}$.

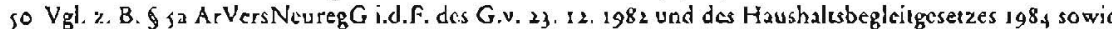
$\$ 2423$, b AFG. Vgl. z. B. BSGE \{3, S. 229 fl. sowie BSG v. 20. 10. $19 \$ 3$ - 7 RAr 17 u. 19/83 - zur Ankundigung von Änderungen im Leislungsbascheid. 
gig gemacht oder in das Ermessen der Verwalrung gestellt. So hängt der Leistungsbezug nunmehr z. B. von dem Urteil der Verwaltung darüber $a b$, ob ein Angehöriger der Pflege bedarf (\$ r 24 rb RVO, 44/s9 AFG) oder die Selbstbereiligung an Leistungen nzumutbar $(\$ 182 \mathrm{a}, 1243 \mathrm{RVO})$ oder eine $*$ Härce ist $(\$ 182 \mathrm{f}$. RVO).

Die in letzter Zeiz hefrig geführe Debatte über den Mißbrauch sozialer Leistungen" droht die Rationalizä des allgemeinen Gesetzes vollends zu beseitigen. So soll mach Ansicht einiger Autoren schon nach geltendem Recht wegen Rechtsmißbrauchs im Einzelfall eine Leistung versagı werden könren's, wie gefordert wird, daß zukünftig Sozialleistungen bei grob fahrlässiger Herbeiführung des Versicherungsfalles verweigert werden sollten", während dic Sozialversicherung bisher allenfalls bei vorsätzlicher Selbstschädigung die Leistung entziehen kann.

Da der Kahlschlag in wichtigen Sozialleistungsbereichen bisher politisch und verfassungsgerichrlicls weitgehend reibungslos abgewickelt werden konnte, lyat sich die aulwendige verfas. sungsrecheliche Diskussion, ob Sozialleistungen nicht den Eigentumsschurz des Ar. ${ }_{14}$ GG genossen 14 , für die eigentlich betroffenen Sozialleistungsempfänger bisher als irrelevan erwiesen. Wern diese theoretische Aufwerung des Eigentumsschurzes allonfalls dient, machi das Minderheitsvotum von Benda und Katzenstein"s deutlich, die die A bwernung der Ausbildungsausíallzeiten in der RV für verfassungswidrig hielten und den Sraat aul das Vorbild des wehrbaren Kaufmanns a verpflichten wollten. Dem Stäe als Kaufmann gegenüberteten kann aber nur, wer eben freiwillig in die RV aufgrund kaufmännischer Kalküle über die Renditc sciner Kapizalanlagen eintritt, nicht aber, wer wic dic abhängig Beschäfrigten aufgrund seiner existenziellen Abhängigkeiı und Unsicherheit zur Mitgliedschaft faktisch und rechrlich gezwungen wird.

\subsection{Verschränkung von Sozialpolitik und Tarifpolitik}

Äußerst ambivalent ist die erscmals mit dem Gesetz über den Vorruhestand auch geserzlich eingeführte Verzahnung von Sozialleistungssystcm und Tarifverragssyscem. Zwar können damir beide Bereiche sinnvoller aufeinander abgestimmt werden. Andererseits vird die Tarifpolitik dadurch staarlich instrumentalisiert, und zwar bei dem Vorruhestandsgeld im Sinnc einer staaclich bewußt gegen dic Gewerkschafuspolitik zur Verkürzung der Wochenarbeitszeit propagierten Verkürzung der Lebensarbcitszeit und der Ausgrenzung älterer Arbeirsloser aus dem Beschäftigungssystem.

\section{Wer ist am stärksten betroffen?}

\subsection{Allgemein dic abbängig Beschäftigten}

Die Leistungskürzungen senkten schon seit 1976 das Einkommen jencr abhängig Beschäftigten, die als Nichterwerbsfähige und -tätige voll auf Sozialleistungen angewiesen sind, während erst ab r 980 die Netto-Löhne der Erwerbstätigen real sanken. Hat das Soziallcistungssystem bisher schon weitgehend Geld nur innerhalb der Bei-

st Vgl. die Beiträge in: Selbsrverantwortung in der Sohidargemeinschafr. Verölf. d. Konrad-AdenaucrStifung, 19S1. Kritisch: Brótz, Dic sog, "Letstungsmißbrauchsdiskussion..., Soziale Sicherheit 1982, S. I $2 \mathrm{fF}$.

s2 So Stcuncr, Schwachstellen der Gesctzgebung im Gesundheitswesen - "Legale W/ege des Mißbrauchs, VSSR 1983, S. Issff.

13 So Ecker, Das scluadensstiftende Verselwulden gegen sich selbst und die soziale Sicherung, in: Selbscuerantworung... (Fn. si), S. ss If.

if Als Ubersicht: Sieveking, Der veriassungstechrliche Eigentumsschutz sozialer Rechtsposizionen, ZiSozRef 1983 , \$. 693 II.

is BVerfGE s8, S. 8, If 
tragszahler der Sozialversicherung und der abhängig Beschäftigten umverteilts, wenn es nicht gar die relativen Einkommensunterschiede zwischen den abhängig Beschäftigten und insbesondere zwischen den abhängig Beschäftigten und den Selbständigen verschärft has '7, so führt insbesondere die Reduktion des Staatszuschusses dazu, daß die Lasten der Krise und ihre Finanzienung noch stärker von den Lohnabhängigen getragen werden müsscn. So sciegen die Belastungen der Arbeiter- und Angesrelltenhaushaice mir öffentlichen Abgaben in dem letzeen Jahrzchnt stärker an als die aller anderen Gruppen der Bevölkerung ${ }^{\S}$. Dadurch, daß ab 1984 die Sonderzahlungen voll in die Beitragserhebung einbezogen werden, werden gerade die unteren und mitzleren Einkommensbezieher, deren Jahrescinkommen unterhalb der Beitragsbemessungsgrenze (1984 DM $5200,-$ in der RV, DM 3900,- in der KV) liegt, verstärkt mit der Finanzierung der Sozialversicherung belastet's.

Nicht nur hierdurch, sondern vor allem auch durch dic ungleiche Vereeilung der Lasten und durch die Tassache, daß die gesellschaftlich besonders diskriminierten Gruppen sehr viel stärker auf staatliche Leistungen angewiesen sind, um überhaupt ansarzweise gleichberechrigue Lebenschancen zu haben, verschärfen die Kürzungen im Sozialleistungssystem die Spaltung innerhalb der abhängig Beschäfrigren und der Bcvölkerung.

4.2. Frauen ${ }^{60}$

Schon die ersten Kürzungen im Sozialleistungssystem, im HaushaltsstrukturgesetzAFG von 1975, trafen vor allem Frauen, da die arbeitsmarktfördernden Leistungen von einer langjährigen vorherigen Berufstätigkeir abhängig gemachc wurden, die Frauen wegen ilhrer (immer noch vorhandenen) stärkeren Belastungen durch die Familie sehr viel weniger vorweisen können als Männer. Genauso wirkten die verschärtten Ausgestaltungen der Anwartschaftszeiten beim Aig, bei den Kuren und bei den vorzeitigen Renten in der RV. Auch das (zudem ab 1984 gekürzte) Mutterschafrsgeld sollte niche nur dazu dienen, es den Frauen zu erleichtern, Erwerbstärigkeit und familiäre Aufgaben miteinander zu vereinbaren, sondern auch dazu, den Frauen wegen familiärer Bindungen den Abgang aus dem Erwerbsleben schmackhaft zu machen. Auch die zahlreichen Kürzungen bei den sozialen Dienscleistungen trafen vor allem Frauen, die einerseirs in diesen Bcreichen überdurchschnirtlich stark beschäftigt sind und andererseits auf sie angewiesen sind, um die Doppelbelastungen von Arbeit und Familie ertragen zu können. Schließlich treffen die Kürzungen der Basisleistungen des Sozialleistungssystems, Alterstenten und Sozialhilke, Frauen besonders hart, da die Armut alter, alleinstehender Frauen groß ist. Wegen der Erhöhung der Anwartschafiszeiten und der Anrechnung von Einkommen der Ehegatren bei der Alhi erhiciten im Seprember $198233 \%$ der arbeirslos gemeldecen Frauen keine Leiscungen der BA gegenüber (nur) $20 \%$ bei den Männern ${ }^{6}$.

\6 Transfar-Enquêre-Kommission (Fn. 35). S. 31 f,,143 f.

s7 So, schr vorsiclitıg: Sozidjstische Siudiengruppen. Brauchen wir den Sozialstaat?, in: Dit Zukunft... (Fn. 7), 5. 17 iff

${ }_{5} 8$ Transfer-Enquêze-Kommission (Fn. 35), S. 149/30: Uncersuchung des DIW, FR v. 28.7. 1983 , S. 6.

is Faupel, Rentenpolsik, Improvisauon stat Srrukturreform, Soztale Sicherhet 1983, S. 172 f., 175.

$60 \mathrm{Vgl}$. Kickbusch/Rıedmuller, Die armen Frauen, $198_{4}$; Gerhard/Metz, Bonn drangt Frauen am Arbeitsmarkt ins Aus, FR 27. 1. 1982.

6) Hans-Bockler-Stifung (Fn. 38), S. 57. 
Selbst ein konservarives wirtschafuswissenschaftliches Inscitur ${ }^{6 x}$ und die Karholische Deursche Bischofskonferenz ${ }^{63}$ kritisierren die Kürzungsaktionen, insbesondere jene im BAföG, Kindergeld und der Sozialhilfe, da sie große Familien übermäßig stark betrafen und der geplante Familienlastenausgleich über das Steverrechr noch nicht einmal diese Kürzungen wieder wettmachen wird.

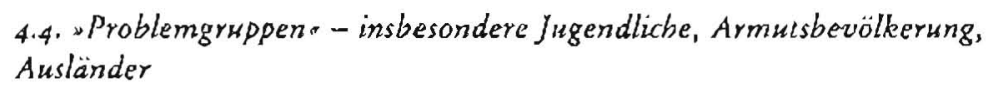

Wie die Nichtanpassung und die Kürzungen der Sozialhilfe vor allem die Armutsbevölkerung und die geringere Anpassung der Renten vor allem die Rentner betrafen, so belasteten die Abschaffung des Schüler-BAföG, die Umstellung des Studenren-BAfoG auf Darlchen, die Kurzungen des Kindergeldes und die Erhöhung der Anwartschaftszeiten bei der Alhi und dem Alg vor allem die Jugendlichen, Berufsanfänger und Ausländer. Die Ausländer sind zudem versrärkı durch die diskriminierende Gestaltung der Verwaltungsverfahren und durch ihren unsicheren Status bei der Arbcitserlaubnis betroffen.

\section{Perspektiven}

Hauptzicl und -crgebnis des Abbaus und Umbaus des Sozialleiscungssystems war es also, mit der Senkung des Markclohns auch den «politischen Lohn «, das Soziaieinkommen, neu und niedriger zu bestimmen, die Lasten möglichst unbemerkr und ungleich zu verteilen und dadurch Ausgliederungs- und Spaltungsrendenzen zu stärken sowie Stracegien und Prozesse der Reindividualisierung und der (angeblichen) Markteffizienz wieder einzuführen. Aber diese Politik stößt auf das Beharrungsvermögen der traditionellen sozialstaatlichen Systeme, auf den Widerstand der von ihr Betroffenen und auf gegenläufige gescllschaftliche Entwicklungen, so daß sie äußersı ambivalent bleiben muß. Ohne der Politik des Sozialleistungsabbaus durch MumaBungen über die objektive Logik der gesellschaftlichen Entwicklung die höheren Weihen der Vernunft geben zu wollen, seien doch cinige dieser zwiespältigen Tendenzen näher analysiert, wie gezeigt werden soll, daßs sich gleichsam whinter dem Rücken * der Kürzungen und ihrer Akteure auch andere Encwicklungen durchsetzen können.

5.1. Immanente Brüche in den klassischen Bedarfslagen der Sozialversicherung Vereinheitlichung des Sozialversicherungssystems?

Zukünftig düften sich die traditionellen und immanenten Brüche in den Bedarfslagen der Sozialversicherung noch verschärfen:

- Dic Arbeisslosenquote wird selbst bci cinem sehr unwahrscheinlichen starken und kontinuierlichen Wirtschaftswachstum weiterhin hoch bleiben und ständig das labile Verhältnis von ArblV - Sozialhilfc - RV wie das Leistungssystem des AFG und die Finanzierung des gesamten Socialversicherungssystems gefährden;

62 Frizsehe, Zu den sozialpolitischen Wirkungen des Haushaltsbegleitgesetzes 1983 , Mitu. des RWI 1983 , S. $187 \mathrm{I}$.

63 FAZ v. 16. 3. 1984 , S. 5, FR v. 18. 3. 1984, S. 4 und FAZ v. $18.4 \cdot 1984$, S. 2. 
- ab 1990 verschlechtert sich das Verhältnis von Beitragszahlem zu Rentnern (sog. Rentenlastquotienc) erheblich ${ }^{6}$, was bei Fortschreibung der jetzigen Aufgaben, Leiscungen und Finanzierungen der RV zu einem RV-Beirrag von über $30 \%$ des Lohns führen müßte:

- nur z. T. können die Lücken dadurch gefüllt werden, daß wegen des sinkenden Jugendlastquotienten (Verhältnis der Jugendlichen uncer 18 Jahren zur Gesamezahl der Erwerbstätigen) Aufgaben und Mittel aus dem Jugendbereich umverteilt werden oder das Rentenbezugsalter wieder auf das 65. oder gar 70. Lebensjahr hinausgeschoben wird.

- Mir der Zunahme der "Alten" an der Bevölkerung wachsen die Aufgaben für die Pflege, dic zwar grundsätzlich, wie z. B. in den Niederlanden, über cinc Pflegeversicherung zu decken sind ${ }^{\text {bs }}$; doch jede Lösung des Risikos „Pflegew wird quer zur zraditionellen Spartenteilung der Sozialversicherung stehen und muß stärker bedarfsoricntiert sein.

- Das gleiche gilt für die anderen noch nicht "gelösten* Probleme der Sozialpolitik: die starke Belascung der Familien und die ungenügende Sicherung der Frauen, die Zunahme von Systemerkrankungen (Herz-, Kreislauf-, rheumacische-, Zuckerkrankheiten etc.) sowie psychiatrisch-psychosomatische Erkrankungen, deren Prävention und Kuration in die Bereiche anderer Versicherungsträger hineinragt (RV, UV) und $z$. T. grundsätzlich das traditionelle Aufgaben-, Leistungs- und Organisationssystem der Sozialversiclserung verläßt (Bewältigung der betrieblichen und allgemeinen gesellschaftlichen Ursachen dieser Krankheiten, Aktivierung der Parienten etc.). Ansätze hierfür sind die verschiedenen Systcme der Verbundfinanzierung von Rehabilitationsleistungen z. B. in der gemeindenahen Psychiatrie oder in der Suchttherapie. Wenn cine komplexc soziale Defizitstrukcur verändert und die Betroffenen selbst akriviert rverden müssen, erwejst sich der traditionelle Verteilungsmodus der Sozialleistungssysteme als inadäquat, der nur individuelle Rechesansprüche gewährt und sie über Vorversicherung (Anwartschaften) und den rein individucll gemessenen Bedarf (Krankenschein, Versicherungsverlauf) vermitrelt und über hoch spezialisiere Dicnstleistungen (Arzt, Krankenhaus etc.) befriedigt.

Schon die systemsprengende Struktur dieser Probleme, wie der allgemeine Zusammenhang zwischen den verschiedenen Sozialleistungssystemen über ihrc Abhängigkeit von den zyklischen und strukturellen Entwicklungen des Wirtschaftsprozesses werden die Incerdependenzen und Flcxibilisierung der verschiedenen Leistungssysteme wachsen lassen. Besteht Aussicht darauf, daß die inkrementalistische Politik des Sichdurchwurscelns, der punkruellen Kürzungen und der Verschiebung von Finanzmassen zwischen den verschiedenen Systemen aufgegeben wird? Eíne solche Politik hat den Vorteil einer politischen Verschleierungstakcik und entspricht jener polisischen Tauschlogik, für die sich die Sicherung sozialer Bedarfe auf die Verschiebung abstrakter Geldmengen reduziert. So dürfee weder sachlich-inhaltlich noch wegen der Form traditioneller Sozialpolirik die Möglichkeit bestehen, die Risiken und Finanzmassen der verschiedenen Sozialleiscungsträger neu zu ordnen - es sei denn die beiden nachfolgend beschricbenen Tendenzen können politischen Neuordnungen Schubkraft verleihen.

64 Beriche über dic Bcvölkerungsentwicklung, BT-Drucks, $10 / 863$ v. s. 1. 1984, S. 53 if.

6) Vgl. die neucren Geseczesinitiaciven der SPD-Hessen FR v, 12.5. 1984, S. 4 und der Grünen FR v. 23. 5. 1984. S. 36 sowie grundsàtzlıch: BMJF, Das Problem der PRegebcdürfigkcit ..., ZiSozRel 1979, S. 106 If.: Berche der Bund-Linder-Arbeitsgruppe $\times$ Aufbau und Finanzicrung ambulanter und stacionärer Pflegedienste" 1980. 
Zwar hat die Krise 1974 ff. allzu deutlich gemacht, daß das Sozialleistungssystem total vom Erwerbssystem, d. h. der Entwicklung des kapitalistischen Wirtschaftsprozesses, der Geschwindigkeit und Struktur der Kapitalakkumulation und der ihr folgenden quanticativen und qualitativen Zusammensetzung der Arbciterbevölkerung abhängt. Es ist und bleibt auch ein wesentliches Ziel aller staatlichen Sozialpolitik, die Reprodukcion der Lohnarbeiter zu sichern und den Lohnarbeiterstatus immer als notwendige und atraktive Alternative zur Nichtarbeit aufzuwerten. Troczdem bestehc selbst im Kernbereich des Sozialleisnungssystems, der SV, zunehmend die Tendenz, rein bedarisbezogene Elemente zu verwirklichen (s. 0. 3.4.). Das ist nicht nur Produkt einer sich sozial gebärdenden Kürzungspolitik, sondern entspricht auch grundsätzlichen Strukcurentwicklungen.

r. Dies hängt einmal mit dens eben analysierten Punkt der zunehmenden immanenten Brüche in den klassischen Bedarfslagen dur Sozialversicherungssysteme zusammen. Ist eine Neuverteilung der verschiedenen Risiken zwischen den unterschiedlichen Organisationen des Sozialleistungssystems nich möglich, so ist es zumindest der kleinste gemeinsame politische Nenner, in Teilbereichen eine Basisversorgung einzuführen ${ }^{66}$, die den ständigen Anpassungsdruck von den differenzierten Spezialsyscemen nimmt. Eine Mindestrente entlaster die Sozialhilfe und erleichtert den Übergang aus der Arbeitslosenversicherung in das Rentensystem. Ein allgemeines und einheitliches System der Pflege und Rehabilitation Behinderter beseitigt den Dauerkonflikt zwischen KV, RV und Sozialhilfe darüber, wer für Pflegefälle zuscändig ist. Wird eine solche Basisversorgung gekoppelt mit einem auf ihr aufbauenden System einkommens- und bcitragsbezogener Leistungen, so sind die zwei gegensätzlichen Prinzipien des Sozialleistungssystems in Einklang gebracht: die bedarfsbezogene Grundsicherung und die Sicherung des Status und der vorgefundenen sozialen Differenzierung. Daß die allgemeine Enrwicklung in den westeuropäischen, kapitalistischen Ländern in diese Richtung weist, hat unlängst Alber in einer systemvergleichenden Studie aufgezeigt ${ }^{67}$.

2. Vor allem aber dürfte diese Entwicklung cinmal die ambivalente Folge jener oben beschriebenen Politik der Kürzung des "politischen Lohns kung der Lohnersatzleistungen sein, die, wie gezeigt, zunehmend dazu führ, daß das System der Basissicherung, die Sozialhilfe, einspringen muß. Auch sind die Kürzungen der Lohnersaczleistungen und Familienleistungen (Kindergeld) für die Betroffenen sozial und vor allem aber für die Gemeinden als Träger der Sozialhilfe finanziell nur noch erträglich, wenn sie nach Bedarfslagen differenziert werden. 3. Zum anderen verbirgt sich dahinter auch cin allgemeiner Trend. In der Zeit von 1960 bis 1980 , und zwar durchweg vor und nach der Krise $1974 \mathrm{ff}$., sind die Sozialleiscungssysreme, die aus dem allgemeinen Staatshaushalt finanziert werden und deren Bezug nach Bedarfsgesichespunkten organisiert wird, die also nicht direkt an das Lohnsystem angckoppelt sind, im Verhältnis zum Wachstum des gesamten Sozialleistungssystems ("Sozialbudget $1960-82$ : $7 \mathrm{fach}$ ) und des Sozialversicherungssystems (1960-82: 8fach) erheblich überproporrional gewachsen: Wohngeld rooofach (wegen Aufhebung der Mietpreisbindung), Ausbildungsförderung 40-, Arbeitsförderung 18-, Sozialhilfe 15-, Jugendhilfe $13^{\text {fach }^{48}}$.

4. Die Ursachen für diese Entwicklung, daß der Anteil derjenigen Bevölkerungs-

66 Zur Diskussion bei der SPD s.o. (Fn. g); bei den Grünen und Altemaciven: Opielka, in: T. Schmid (Hrsg.), Befreiung von falscher Árbett. Thesen zum garantienen Mindesteinkommen, 1984.

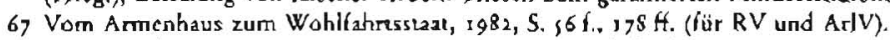

68 Gesellschalu. Dacen 1979 (Hrsg. BMA), S. 259 u. Sozialberiche 19\$3, S. 581. 
gruppen, die überwiegend von Sozialleistungen lebcn, ständig wächst und zu Beginn der Boer Jahre in der Bundesrepublik erstmals die Zahl der Arbeiter übertrafon, können nur angedeutet werden:

- Das auf dem Arbeitsmarkt realisierte und individuell verfügbare Lohnvolumen reicht immer weniger dazu aus, das sich entwickelnde Reprodukrionsniveau der Arbeirskraft (die wordentliche« Ausbildung der Kinder, die Pflege im Alter) sicherzustellen; scaacliche, kompensatorische Leistungen werden norwendig.

- Während die Anforderungen an die Herstellung und Reproduktion der individuellen Arbeitskratt wie der Arbeirskräfte insgesamr wachsen (veränderte Arbeitsbedingungen, psycho-sozialer Verschleiß der Arbcitskräfze, Mobilitär der Arbeirskräftc, ständig veränderte Qualifikationserfordernisse), verlieren dic Bereichc der "natürlichen*, privaten Reproduktion in der Kleinfamilie und den kleinen Gemeinschaften immer mehr an Bedeutung und Leistungsfähigkeit. Dadurch werden die Reproduktionsaufgaben zunehmend vergesellschafter und von der kapitalistischen Produktionsweise (marktmäßig vermittelte personale Dienstleistungen, Großverbände und Interessenorganisationen) sowic von den staatlichen Steuerungs- und Dienstleistungsaktivitäten erfaßr ${ }^{70}$. Die staatliche Finanzierung der Reprodukrionsleistungen über das Steuersystem sowie die Gewährung öffentlicher sozialer Dienstlcistungen der Reproduktion (z. B. Bildungs- und Rehabilicationswesen) sind aber schon von ihrer Struktur her bedartsbezogen, ihre Verbindung mit der Lohnarbeir und industricllen Produktion ist schr vermittelt, ihre Rückverlagerung in die Kleinfamilic dürfte sachlich nur begrenzt möglich scin - was z. B. die neue Diskussion um die Revision der BAföG-Kürzungen deutlich macht ${ }^{71}$.

- Verringertc sich früher der Bevölkerungsanteil der abhängig Beschäfrigten durch die längere (schulische) Ausbildung sowie Umschulungs- und Requalifikarionsaktivitäten und dic frühe Verrentung, so wird dic Arbeic nunmehr durch die internationale Arbcitsteilung und zunehmende Rationalisierungsmóglichkeicen insgesamn weniger. Selbst eine radikale Neuverteilung der Arbeit durch Senkung der Lebens-, Jahres-und Wochenarbeitszeit muß den Reproduktionsbereich aufwerten, während der andere Lösungsweg, die Arbeitskrälte in die Arbeitslosigkeit zu entlassen, crst reche große Teile der Bevälkerung in die nicht mehr oder nur noch locker an das Beschäftigungssystem gebundenen Sozialleistungssysteme oder in alternative Lebens-, Produktions- und Reproduktionsformen abdrängt. Grundsätzlich wird also das Verhältnis zwischen der Sphäre der Arbeit und der Sphäre der Nicht-Arbeit (i.S. nicht frendbestimmter, nicht rein ökononisch determinicrer Arbeit) neu bestimmt und gewinnt die Nicht-Arbeit an gesellschaftlicher Bedeutung'². Das Versicherungsprinzip, das in Finanzierung (prozentualer Bcicrag vom Lohn) und Leistung (Lohnersatzleistung, Rehabilitation des Arbeitsvermögens) ganz auf den Lohnarbeiterstatus und die relacive Höhe des Lohns sowie die besondere, individuellc Leistungsfähigkeit des Lohnarbeiters ausgerichtet ist, war in der Frühphase des Sozialleistungssystems norwendig, um das System der Lohnarbeit gegenüber den überholten Formen vorindustrieller Arbeit abzusichern und der Verallgemeinerung der Lohnarbeit Rechnung zu tragen. Nunmehr verliert dieses Prinzip mit der Ausdehnung der Sphäre der $n$ Nicht-Arbeita und der zunehmenden Vergesellschaftung der Reproduktion außerhalb des Bereichs und den Formen der Lohnarbeit an Legi-

69 Alber, ebenda, S. 209 m.w.N.

70 Ausführlicher hieczu Mückcnberger, Thesen zur Funktion und Enewicklung des Soxialrccht5, KJ 1976, S. $337 \mathrm{fl}$.

71 V Gl. FAZ v. 6.4.1984, S. s.

7. Vgl. au diescm Prozeß: A. Gork, Wege ins Paradies, 1983; BonB/Hcinzc, Arbcil, Lohnarbeit ohne Arbeis, in: dies., Arbeitslosigkeit in der Arbeiesgesellschale. 1984, S. 7 ff. m.w.N. 
umationskrafe und ist es immer weniger fähig, für große Teile der Bevölkerung die existentiell wesentichen Leistungen zu sichern.

Insoweit entspricht die oben (3.4.) analysierte Auflösung des Versicherungsprinzips zwar einem langfristigen Strukturwandel, dies aber in höchsr ambivalenter und gesellschaírspolizisch gefährlicher Form. Denn wie diese notwendige scarke Orientuerung des Sozialleistungssystems an Bedarfsprinzipien politisch verarbeiter wird, ist offen. Um Entsolidarisierungen und weicere Spaltungen zu verhindern und den Druck der industriellen Reservearnee auf die (noch) Arbeitenden abzumildern, wäre es notwendig, eine Mindestsicherung Aller ohne kontrollierende und diskriminierende Vergabepraktiken durchzuseczen ${ }^{7 s}$. Jedoch geht die Kürzungspolitik den entgegengesetzten Weg, indem die Nichtertverbstätigen aus dem Sozialversicherungssystem ausgegrenzt und die Entsolidarisierung und Spaltung der Bevölkerung vorangetricben werden, wic die zunehmende Einführung von Bedarfsprinzipien gerade auch in Kernbereichen der SV dazu dient, den erreichten Stand von Glcichheit und materieller Sicherheic, der sich bei abhängig Beschäfrigten auch am vorherigen Lohn orientieren muß, abzubauen und die soziale Kontrolle zu erhöhen.

5.3. Krise der Produktionsformen des Sozialleistungssystems: Reindividualisierung und soziale Kontrolle - Selbstbilfeberuegung

In der sozialpolitischen Diskussion ist gerade in den letzten Jahren herausgearbeitet worden, daß das Sozialleistungssystem auch wesentliche Funktionen der sozialen Kontrolle* hat, insbesondere die ständigc Ein- und Anpassung der abhängig Beschäfrigten an das System der Lohnarbeir ${ }^{74}$. Es sind gerade dic Mechanismen "sozialer Kontrolle", die in der Krise verschärit worden sind: Der Druck auf die Arbeitslosen und Sozialhilfeempfänger, jede Arbeit anzunehmen und sich den veränderten Marktbedingungen anzupassen, dic hoheitlich-administrativen Kontrollen der Bedarfsaspekre in der SV, die die Einheitlichkeit und Kollektivitär der Risiken in individuelle Norlagen auflösen, sowie die Selbstbeteiligung, die ökonomische Kalküle durchsctzen soll.

Durch diese drei Mechanismen werden die schichtenspezifische ungleiche Inanspruchnahme der Sozialleistungen noch verstärkt und zugleich die allgemeinen gesellschafelichen Ursachen der Risiken Arbeitslosigkeit und Krankheic zu individuellen sowic individuell und administrativ sceuerbarem (Fehl-)Verhalten umgewcrtet.

Generell zerstört die Senkung der Lohnersatzlcistungen den Solidarausgleich zwischen den abhängig Beschäftigten. Die sclektive Wirkung der Kürzungen verstärkt und reproduziert zudem die gesellschaftliche Spaltung und Konkurrenz der Arbeitnehmer in Arbeitsplatzbesitzer und Nichtbesitzer, Männer - Frauen, Stamm- Randbelegschaft, Ausländor - Einheimische.

Grundsärzlich ist es vor allem Aufgabe der Gewerkschaften, auf betrieblicher und gesamtgesellschafelicher Ebene die Solidarität im Handeln und im Bewußrsein der Arbeiznehmer herzustellen und zu stärken. Nur sind die Gewerkschaften von ihrer Organisationsscruktur (betriebliche Basis) und ihren Handlungsinstrumenten (Tarifvertrag, Sireik, Mitbestimmung) sowie den Inhalten ihrer Politik her vor allem

73 Vgl. dic Bciträge bei T. Schmid (Hrsg.) (Fn. 66) sowie Olfc, FR v. 23.8. 1983, S. 10 und Redaktion der Widcrspriche, FR v. 28. 3. 1984.

74 Vgl. Rödel/Guldimann, Sozialpolirik als soziale Kontrolle, Stamberger Studien 2, 1978. S. i If.; Lenhardu/Offe, Sisatstheoric und Soxialpolitik..., in: v. Ferber u. .. (Hrsg.), Soziologie und Sozialpoliuk, 1977, S. $98 \mathrm{ff}$. 
auf dic Erwerbstätigen und den Becrieb als Handiungsfeld konzentriert"s. Ihre Handlungsinstrumente erfassen zwar die Basis des Sozialversicherungssystems (Lohn) und wichnige Randbereiche des Sozialleistungssystems (tarifliche Zulagen bei Arbeiesiosigkeit, Rationalisierungsschurz, Betriebsrenten, Arbeits- und Gesundheitsschutz im Berrieb, Beteiligung an der Selbstverwaltung der Sozialversicherung). Sie können jedoch die trocz aller Verschränkungen weiterhin bestehenden Diskrepanzen zwischen Produktion und Reproduktion und deren jeweils verschiedenen Vergesellschaftungsformen (Tausch auf dem Arbeirsmarkt und betriebliche Herrschaft/Gegenwehr - Sozialversicherung, Familie, allgemeine politische Verkehrsformen) nur teilweise überwinden. In der staatlich vermittelten Sozialpolitik wie in den Bereichen nichtbetrieblicher Reproduktion und Lebensweisc (Familie etc.) sind die Gewerkschaften allenfalls eine Interessengruppe uncer anderen. Beispielhaft seien hier die Schwicrigkeiten einer eigenständigen gewerkschaftlichen Arbeitslosenpolitik $^{76}$ oder der geringe gewerkschaftliche Einfluß auf die Sparpolitik der SPD-FDPRegierung oder die Schwierigkeiten genannt, über den Tarifvertrag Fragen der beruflichen Bildung oder der Ergänzung der Sozialleistungen zu regeln.

Hier liegt nun gerade eine der wesentlichen Funkrionen der Selbsthilfebewegung (Frauenhäuser, medizinische Selbsthilfcgruppen, Arbeitsloseninitiaciven, Jugendinitiariven, Kinderhäuser, Instandbesetzer etc. $)^{77}$. Gerade in ihren gemeinsamen Merkmalen: Bedürfnisorientierung. Identität von Trägern und Nutznießern der Leistung, geringe Professionalisierung, basisdemokratische und unbürokratische Orientierung, haben sic einen zentralen Modus des staatlichen Sozialleistungssystems verändert: Statt passives Objekt verrechtlichter und bürokratisierter privater Unternehmen des sozial-gewerblichen Komplexes (Arzt, Pharmaindustrie cte.) oder öffentlich-rechclicher Verreilungs- und Versorgungsinscitutionen und ihrer sozialen Koncrollmechanismen zu sein, erlangen die Betroffenen ein stärker instrumentelles Verhältnis zum Sozialleistungssystem, das sie mit ihren Bedürfnissen konfrontieren und anhand ihrer Interessen kontrollieren. Die Selbsthilfezusammenschlüsse bilden eine erste kollektive Organisation, um zumindest jene Lebensbereiche zurückzugewinnen, die außerhalb des Einflußbereichs der kollektiven und solidarischen Organisation im Produktionsprozeß stehen, und die nach dem heutigen Stand der Entwicklung sozialer und politischer Kompetenzen in der Bevölkerung unnörigerweise noch bei öffendich-rechtlichen/privaten Institutionen verblieben sind.

Insbesondere wenn die staatlichen Strategien der Instrumentalisierung der Selbsthilfebewegung als Puffer für den Abbau sozialer Dienseleistungen abgewehr werden können, der autonome Bereich sich stabilisieren und intern vernetzen sollte, der Übergang zwischen dem autonomen und dem traditionellen/professionellen Sektor durch Reduktion der Wochen- und Lebensarbeitszeit freiwillig möglich ist und sich verallgemeinert und beide Bereiche sich organisatorisch und finanziell enger miteinander verzahnen, dürfte sich hier ein bleibender und entscheidender Strukturwandel in System der Sozialpolitik herausbilden.

7s Zu dieser Dissoziation von Zielprojekcion und Kamplpotencial der Gewerksehaften gerade in der Sozialpolitik: Muckenberger (Fn. 70), S. 347/8.

76 Vol. Moller-Lucking. Gewerkschafien und arbeieslose Arbeitnehmer, Soziale Sicherheii 1982, S. 33 ff.

$77 \mathrm{Vgl}$ allgemein zur Selbsthilfebewegung: Beyw1/Brombach, Neue Selbstorganisationen, Aus Politik und Zeitgcschicher, B $11 / 84$, S. 1 ff. sowic Deimer u. a., Selbsthille in der Sozialpolitik, Aus Politik und Zeitgeschichrs. B $34 / 83$, S. ${ }_{4}$ ff., beide m.w.N. 

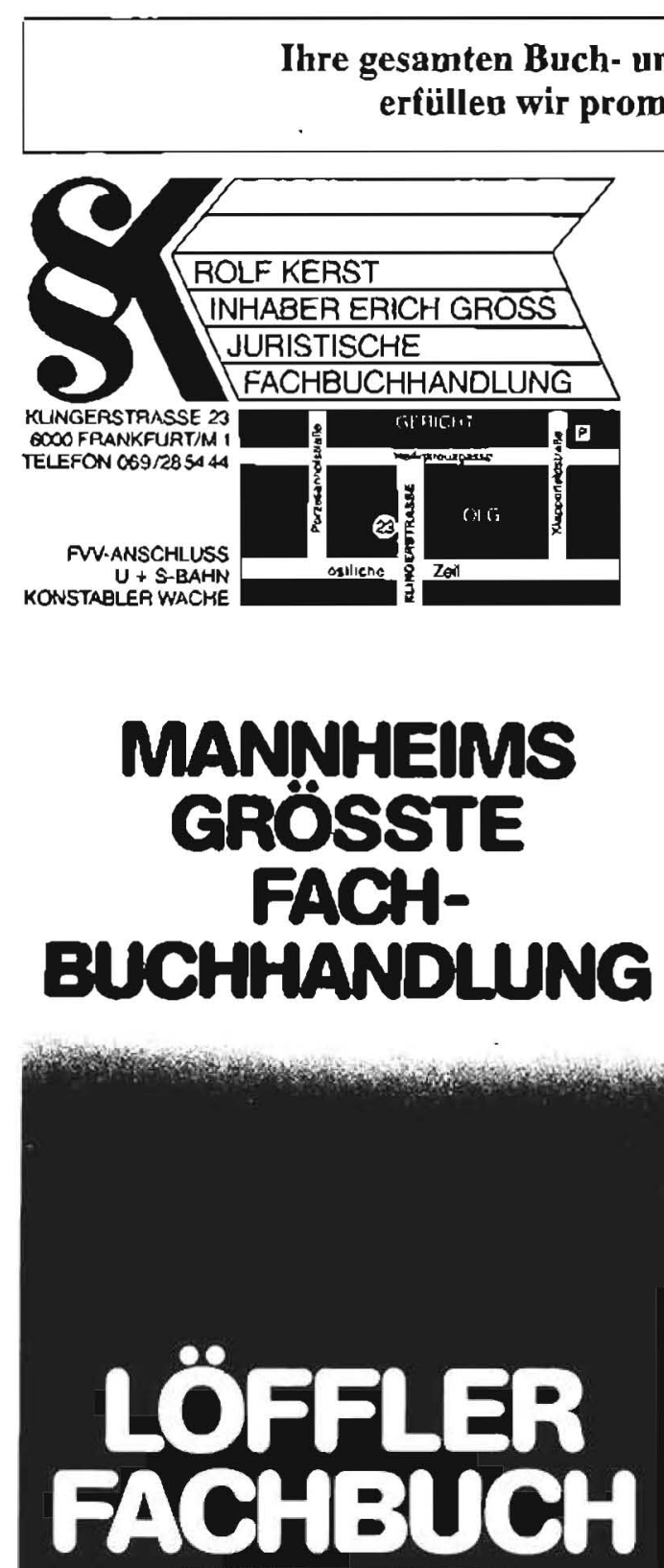

6800 Mannheim $1 \cdot B 1,5$

Breite Straße, Nähe Schloß - Tel.0621/289 12

\section{Buchhandlung \\ KARL KAMLOTH}

Juristische Fachliteralue

lü Sludium und Praxis

Im Neuen Gerichesgebăude

2800 Bremen 1

Telefon 321170

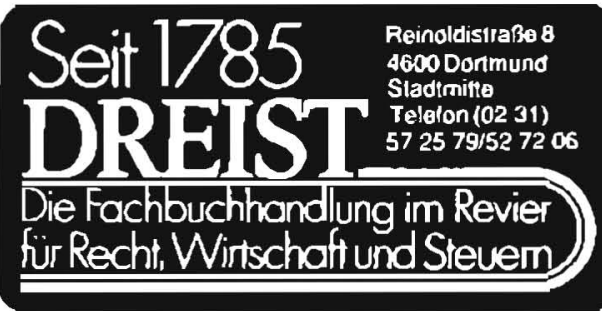

Schŏningh gosata

B UCHHANDLUNGEN platz und im Universitats $\begin{array}{ll}\text { Führend in Wirtschafts- und } & \text { gelände } \\ \text { Rechtswissenschant } & \text { am Hubland }\end{array}$

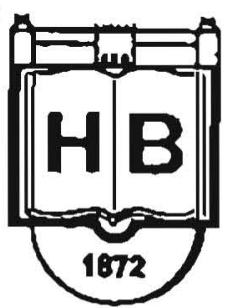

Juristlsche Fachbuchhandlung

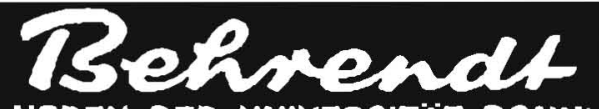
NEBEN DER UNIVERSITAT BONN

Am Hof $5 a \cdot$ Telefon $858021-24$ und Filiale in der Mensa, NassestraBe 11
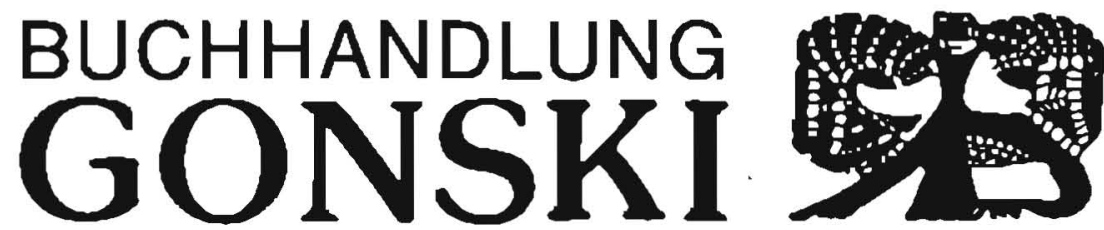

KÖLN · NEUMARKT 24 · TEL. 210528

Fachabte1lung: Recht - Wirtschaft - Steuer Gertrudenstr. 75000 Röln ] Telefon: $0221 / 214284$ 
Ihre gesamten Buch- und Zeitschriftenwuinsche erfüllen wir prompt und zuverlässig

\section{SEIT T 1899 \\ HOSER'S BUCHHANDLUNG STUTTGART}

Die führende Fachbrichhapdlung fuir Reches-, Wirtschafis- und Sozialwissenschafter

Unsere Leis1ungen: Eingehende Fachberatung durch crfahrene Fachbuchhändler Sorortige Lieferung aller wichtigen Fachitel -

auch unverbindlich zur Ansich Schnellste Beschaffung aller anderen, lieferbaren Publikatione Zuverlässige Abonnementsbeueuung für Fachzcischriflen

Erginzungstiefcrungen. Fortsetzungswerk Auf Wunsch regelmäßige Zusendung von Fachbuchinformationen

Schneller Versand Bücher und Grundwerke portofrei.

Hoser's Buchhandlung - Charlouenplav. 17 - 7 Surugarl I Tcl. $0711 / 221656$

\begin{tabular}{|l|}
\hline \\
\hline
\end{tabular}
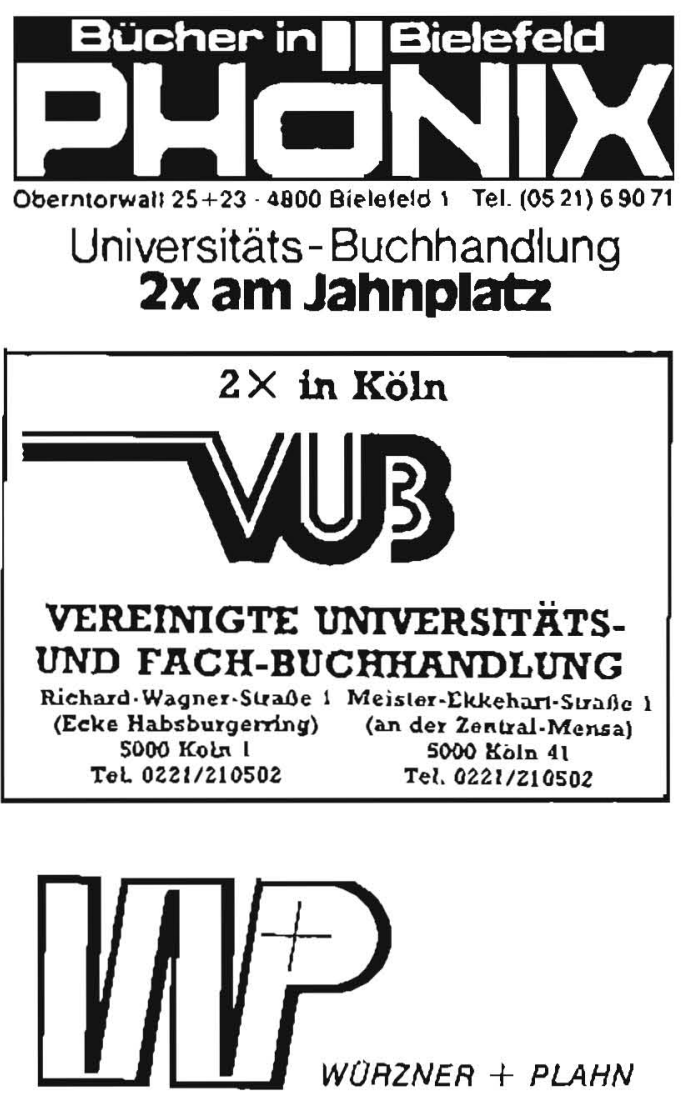

Fachbuchhandlung lür Recht und Stevern

Holtzendorlfstr. 18, 1000 Berlin 19.

Tel. (030) 3235021

Thre juristische Fachbuchhandlung in HEIDELBERG

Recht - Wirtschalt - Steuern für Wissenschaft, Praxis und Studium.

Alle Fachbicher, Zeitschrilten, Entscheidungen, Loseblatlwerke mit Ergänzungslielerungen kastengünstig, zuverlässig und schnell.

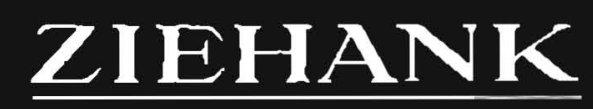

Universitäts buch hand I ung Inh. Walter Torka

6900 Heidelberg 1

Postfach 106266 . Universitätsplałz 12 \& (062-21) 10081-83 Tx. 461754 